\title{
Ornamental Bee Plants as Foraging Resources for Urban Bees in Southern Brazil
}

\author{
Denise Monique Dubet da Silva Mouga, Vanessa Feretti, Jeniffer Cristine de Sena, \\ Manuel Warkentin, Andressa Karine Golinski dos Santos, Carolina Lopes Ribeiro \\ Bee Laboratory, Department of Biological Sciences, University of the Region of Joinville, Joinville, Brazil \\ Email: label@univille.br
}

Received 28 February 2015; accepted 17 March 2015; published 20 March 2015

Copyright (C) 2015 by authors and Scientific Research Publishing Inc. This work is licensed under the Creative Commons Attribution International License (CC BY). http://creativecommons.org/licenses/by/4.0/

(c) $\underset{\mathrm{EY}}{\mathrm{B}}$ Open Access

\section{Abstract}

Ornamental plants are distinguished by the shape and color of their leaves/flowers and their use in landscaping and gardening. In urban areas, their presence is relevant for the maintenance of bees. Aiming to list the ornamental bee plants species occurring in Santa Catarina State (SC), southern Brazil, a survey of forage resources for bees, recorded in the State, was performed. We refer all published works developed in SC (resulting in the period 1983-2014) which include data from reports of scientific research, monographs, dissertations, Masters Dissertations, Doctoral Thesis, annals of scientific events, scientific articles and books. From the 28 ensuant works, we listed, as ornamental bee plants occurring in SC, 201 species, of 156 genera and 66 botanical families. Of these species, 92 are native (N), 26 endemic to Brazil and 109 exotic (E). Families with more ornamental bee species are: Fabaceae (22 species), Asteraceae (20), Solanaceae (11), Rosaceae (10) and Lamiaceae (8). The most cited species are: Lantana camara $\mathrm{L}$. (N) (Verbenaceae) (9 quotes), Aloysia virgata (N) (Ruiz \& Pav.) Juss. (Verbenaceae) (8), Hedychium coronarium J. Koenig (naturalized) (Zingiberaceae) (6) and Brachyscome multifida (E) (Asteraceae) (5), regarding habitus, herbs $(48.5 \%)$ predominate, followed by bushes $(21.5 \%)$, trees $(16.5 \%)$ and vines $(10.5 \%)$. Preferred colours of flowers by bees are, in decreasing order: white, yellow, red/pink, and others. The genus Ipomoea, Calliandra, Passiflora, Prunus and Senecio have more ornamental bee plant species. A large botanical diversity arises, demonstrating the possibilities for urban bee foraging and ornamental featuring, in a perspective of sustainability.

\section{Keywords}

Apidae, Bees, Floral Resources, Pollination, Urban Fauna 


\section{Introduction}

Crops depend on pollination to have viable economic returns. Apis mellifera is the most widespread pollinator in the world, however, this species is nowadays affected by CCD (Colony Collapse Disorder) [1]. It has been hypothesized that pollination will depend increasingly on native bees for the transfer of plant gametes [2]. Native bees live spontaneously in natural areas but also in urban areas [3]. Natural areas are shrinking worldwide in view of human interventions in the environment and it has been observed that urban areas have been progressively occupied by populations of non-domesticated species, thus turning into havens for wildlife [4]. It is known that crops have higher yields if they are contiguous to areas of native vegetation cover that act as sources of pollinators [5]. In urban areas, pollinators exploit existing open areas (gardens, orchards, squares, parks, sports fields, clubs, vacant lots, vacant areas, etc...) where there are flowering plants of ruderal, ornamental, fruit trees, vegetables, weeds and others species, of varying sizes and habits [6]. Thus, it becomes relevant, in urban areas, to have bee plants for maintaining the diversity of bees. The goal of offering more floral resources for bees in urban areas can be achieved by encouraging the growing of ornamental bee plants, in a perspective of gardening, landscaping and sustainability [7]. Plants known as ornamental are distinguished by the attractive shapes and/or colors of their leaves and/or flowers and are part of numerous groups of cultivated and wild species, including representatives of various botanic families [8]. They are often cosmopolitan and originating from different countries [9].

Santa Catarina (SC) is a state in southern Brazil, with 95,400 $\mathrm{km}^{2}$ of surface (an area equivalent to Austria, Hungary, Ireland or Portugal), subtropical-temperate (including frosts and eventually snow in winter), which shows a strong relief in the form of an increasing altitudinal gradient in an east (Atlantic Ocean)—west (border with Argentina) direction (750 km spacing), with 77\% of its territory above $300 \mathrm{~m}$ altitude and $33 \%$ above 600 $\mathrm{m}$, with a maximum altitude of $1822 \mathrm{~m}$ (Morro da Igreja Mountain), vegetation cover with the Atlantic Forest biome (rain forest), strong agricultural production (cereals, temperate fruit orcharding) [10]. Although it is the second largest exporter of honey from Brazil (coming from colonies of Apis mellifera), the state has suffered with the CCD [11].

Previous surveys of bee species and works related to bees have been done in SC and included lists of bee plants as their floral resources, forming a mass of information about bee-flower relationships. However, none of them was directed to the observation of foraging of bees on ornamental plants. These works gave rise to the question about whether there are ornamental bee plants cited amidst all the papers and, more specifically, of whether type are these plants in terms of habitus, flower color and geographical origin. The aim was to enroll particular ornamental plants, aesthetically pleasing, suitable for gardening and landscaping, to be used, in urban areas, as a draw and food resource for wild bees. This was thought in a view of making it possible for ecologists, farmers, plant enthusiasts and gardeners, to enhance the urban environment as a biological corridor, connecting nearby forest fragments.

\section{Material and Methods}

The work was done during the year of 2014. The analyzed papers compose the library of the LABEL-Bee Laboratory of the University of the Region of Joinville. The period covered by the papers runs from 1983 to 2014. The works include academic data (scientific initiation reports, monographs, Masters dissertations, Doctoral thesis), technical reports, proceedings of scientific events, scientific articles, book chapters and books. We selected 28 works considering the following criteria: the work had been done in Santa Catarina State and the subject was about bees in any of the following themes: bee rearing, surveys of species, pollination studies, palinology of bee plants, bee pollinators or related ones. The procedure for the selection of ornamental bee plants of Santa Catarina State followed the steps described hereafter (Figure 1). After compiling all the works, we searched for the theme bee plants in each paper. All the plants mentioned in each paper were listed in a database. The habitus, color of flower/inflorescence, geographical origin and ornamental condition of the species were looked for and digested. The ornamental plants were then selected from the database to constitute the list of ornamental bee plants occurring in SC State.

\section{Results and Discussion}

We listed, from the 28 selected works, 956 bee plant species for SC, from which we verified, as ornamental ones, 

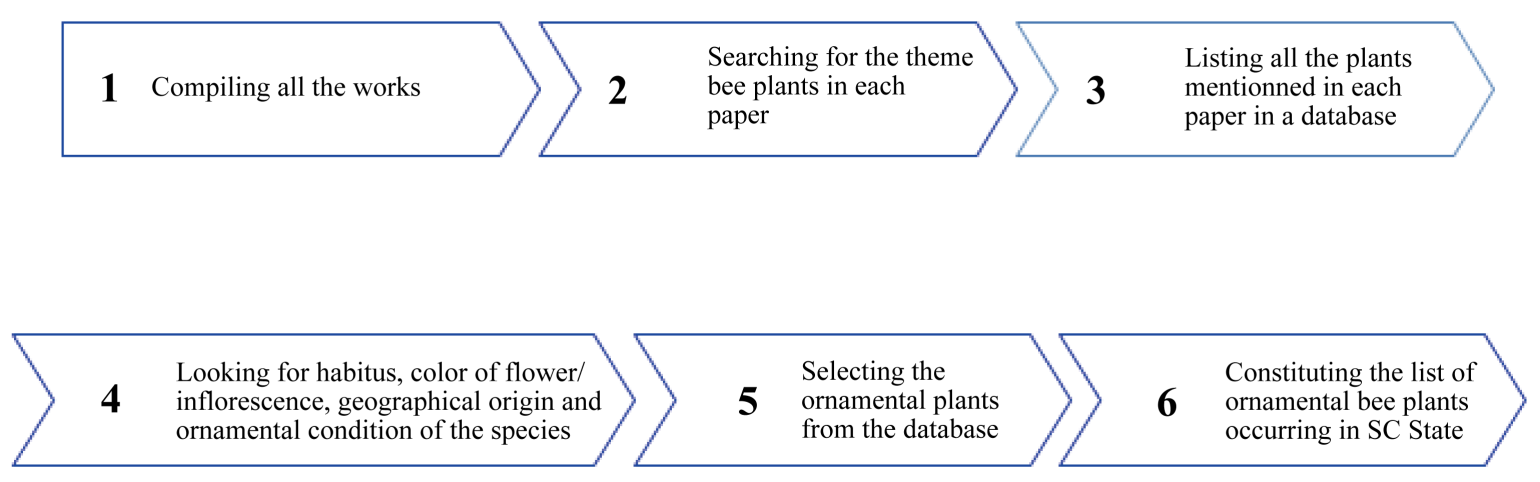

Figure 1. Flow chart of selection of ornamental bee plants of Santa Catarina State.

201 species, 156 genus and 66 families (Table 1). In urban areas in southern Brazil, [12] reported 64 bee species, [13] 85, [14] 154, [15] 69 and [16] 42. The percentage of ornamentals found in this work constitute $21.02 \%$ of the bee plant species mentioned for SC. No information about this percentage for Brazilian studies was in literature.

The family that shows more ornamental bee plant representatives is Fabaceae with 22 species, followed by Asteraceae (20), Solanaceae (11), Rosaceae (10), Lamiaceae (8), Verbenaceae and Bignoniaceae (7), Cactaceae and Malvaceae (6), among others (Figure 2). [14] mention Fabaceae as the the most represented bee plant species as well as [16]. [17], [18] and [19] found that the three botanical families with more bee plant species were Asteraceae, Myrtaceae and Fabaceae and [20] mention that the the two most attractive plant families to bees were Asteraceae (which provide pollen and nectar) and Lamiaceae (which provide nectar). Fabaceae is a very large family in tropical and subtropical South American habitats, with 2801 species in Brazil [21] and is very visited by bees, the flowers often shaken to free the pollen out of the porate anthers (buzzing) [22]. Asteraceae totals 2064 species in Brazil [23] and shows a floral display of the open type, much sought after by bees because of the ease to land, the conspicuousness of the flowers in the wild and the arrangement of the inflorescences opening in sequence, offering new supplies every day [24]. Solanaceae has greatest diversity of species in South America and Central America, with approximately 470 species in Brazil [25] and its flowers also pass by sonication to have its pollen freed by bees [26]. The other families are reported as having also species attractive to bees [27].

The genera with more ornamental bee plant species are Ipomoea, Calliandra, Passiflora, Prunus, Senecio and Tibouchina with 4 species each one and Acacia, Aloe, Begonia and Rosa with 3 (Figure 3). The differences in ornamental plant presence and abundance are important variables (suggesting different gardening practices, plant availability and selection) that can greatly influence bee populations by determining the overall amounts of their preferred floral resources [28]. In this way, [16] reported 12 botanical families represented by only one species while [14] mention 30 families with only one species. It must be remembered for gardening in a bee friendly purpose that botanical diversity of ornamental plants is a major fact to attend a bigger diversity of bee species.

In relation to the habitus of the plants, in descending order of frequency, are herbs (48.5\%), shrubs (21.5\%), trees (16.5\%), vines (10.5\%) and sub shrubs (3\%) (Figure 4). According to [29], ornamental plants, in terms of biological properties and agrotechnical requirements, are divided into several groups such as trees, shrubs, perennials, biennials, annuals, grasses and bulbs and their use in beekeeping requires research work for being widely recommended. As shrubs and herbs are the kind of plants more often used in gardening [9], both trends go in the same direction, enhancing the possibilities of implementing bee friendly gardens.

In terms of color of the flowers, predominate, in descending order, the white flowers (69 species), yellow (30), red (26), pink (25), lilacs (15), blue (15), orange (10), purple (9) and green (2) (Figure 5). These findings follow the general tendencies of flower color preferences of bees (bright white, yellow, blue or violet or those that reflect ultraviolet light), described by authors such as [30] and [31], among others. As the color of the flowers have, many times, some relation to the botanical family they belong to [27], the knowledge of the bees' preferences for these taxa should be in mind, in landscaping practices aiming succession of colors in gardens following the seasons. 
Table 1. List of ornamental bee plants in Santa Catarina State, southern Brazil. References of this table are after the table.

\begin{tabular}{|c|c|c|c|c|c|c|c|c|c|c|}
\hline $\mathrm{N}$ & Family & Genus & Species & Author & $\mathrm{N}$ & End & $\begin{array}{l}\text { Exot./ } \\
\text { introd }\end{array}$ & Hab & Col & References \\
\hline 1 & Acanthaceae & Justicia & brandegeeana & Wassh. \& L.B. Sm. & 0 & 0 & 1 & S & $\mathrm{R}$ & 27 \\
\hline 2 & Acanthaceae & Ruellia & elegans & Poir. & 1 & 0 & 0 & SS & $\mathrm{R}$ & 27 \\
\hline 3 & Acanthaceae & Thunbergia & erecta & $\begin{array}{l}\text { (Benth.) T. } \\
\text { Anderson }\end{array}$ & 0 & 0 & 1 & S & $\mathrm{L}$ & 20 \\
\hline 4 & Acanthaceae & Thunbergia & grandiflora & Roxb. & 0 & 0 & 1 & V & B & 20 \\
\hline 5 & Adoxaceae & Sambucus & nigra & $\mathrm{L}$. & 0 & 0 & 1 & S & $\mathrm{W}$ & 19 \\
\hline 6 & Aizoaceae & Lampranthus & productus & N. E. Br. & 0 & 0 & 1 & $\mathrm{H}$ & PI & 25 \\
\hline 7 & Amaranthaceae & Alternanthera & brasiliana & (L.) Kuntze & 1 & 0 & 0 & $\mathrm{H}$ & $\mathrm{W}$ & 17 \\
\hline 8 & Amaranthaceae & Alternanthera & dentata & $\begin{array}{l}\text { (Moench) Stuchlik } \\
\text { ex R.E. Fr. }\end{array}$ & 1 & 0 & 0 & $\mathrm{H}$ & G & 17 \\
\hline 9 & Amaranthaceae & Celosia & cristata & $\mathrm{L}$. & 0 & 0 & 1 & $\mathrm{H}$ & $\mathrm{R}$ & 27 \\
\hline 10 & Amaranthaceae & Gomphrena & canescens & R. Br. & 0 & 0 & 1 & $\mathrm{H}$ & PI & 17 \\
\hline 11 & Amaranthaceae & Gomphrena & demissa & Mart. & 1 & 1 & 0 & SS & $\mathrm{W}$ & 17 \\
\hline 12 & Amaryllidaceae & Crinum & americanum & $\mathrm{L}$. & 1 & 0 & 0 & $\mathrm{H}$ & $\mathrm{W}$ & 2 \\
\hline 13 & Apiaceae & Eryngium & ebracteatum & Lam. & 1 & 0 & 0 & $\mathrm{H}$ & $\mathrm{P}$ & $\begin{array}{l}10,11, \\
23,24\end{array}$ \\
\hline 14 & Apiaceae & Eryngium & eburneum & Decne. & 1 & 0 & 0 & $\mathrm{H}$ & W & 3 \\
\hline 15 & Apiaceae & Petroselinum & sativum & Haffn. & 0 & 0 & 1 & $\mathrm{H}$ & W & 17 \\
\hline 16 & Apocynaceae & Catharanthus & roseus & (L.) Don & 0 & 0 & 1 & S & PI & 19 \\
\hline 17 & Apocynaceae & Tabernaemontana & hystrix & Steud. & 1 & 1 & 0 & $\mathrm{~T}$ & W & 2,17 \\
\hline 18 & Araceae & Zantedeschia & aethiopica & (L.) Spreng. & 0 & 0 & 1 & $\mathrm{H}$ & W & 7 \\
\hline 19 & Arecaceae & Syagrus & romanzoffiana & (Cham.) Glassm. & 1 & 0 & 0 & $\mathrm{~T}$ & $\mathrm{Y}$ & 5,21 \\
\hline 20 & Asparagaceae & Dracaena & marginata & Hort. & 0 & 0 & 1 & S & $\mathrm{W}$ & 12 \\
\hline 21 & Asparagaceae & Cordyline & fruticosa & (L.) A. Chev. & 0 & 0 & 1 & $\mathrm{~S}$ & PI & 7,13 \\
\hline 22 & Asteraceae & Acanthospermum & australe & (Loefl.) Kuntze & 1 & 0 & 0 & $\mathrm{H}$ & $\mathrm{W}$ & 4 \\
\hline 23 & Asteraceae & Achillea & millefolium & $\mathrm{L}$. & 0 & 0 & 1 & $\mathrm{H}$ & $\mathrm{W}$ & 25 \\
\hline 24 & Asteraceae & Bellis & perennis & $\mathrm{L}$. & 0 & 0 & 1 & $\mathrm{H}$ & $\mathrm{W}$ & 13 \\
\hline 25 & Asteraceae & Bidens & laevis & $\begin{array}{l}\text { (L.) Britton, Sterns } \\
\text { \& Poggenb. }\end{array}$ & 1 & 0 & 0 & $\mathrm{H}$ & $\mathrm{Y}$ & 4,22 \\
\hline 26 & Asteraceae & Bidens & sulphurea & Sch.Bip. & 0 & 0 & 1 & $\mathrm{H}$ & $\mathrm{O}$ & $7,8,17$ \\
\hline 27 & Asteraceae & Brachycome & multifida & DC. & 0 & 0 & 1 & $\mathrm{H}$ & $\mathrm{L}$ & $\begin{array}{c}18,20,21, \\
23,25\end{array}$ \\
\hline 28 & Asteraceae & Calea & uniflora & Less. & 1 & 0 & 0 & $\mathrm{H}$ & $\mathrm{Y}$ & 22 \\
\hline 29 & Asteraceae & Calendula & officinalis & $\mathrm{L}$. & 0 & 0 & 1 & $\mathrm{H}$ & $\mathrm{Y}$ & 18 \\
\hline 30 & Asteraceae & Centaurea & cyanus & L. & 0 & 0 & 1 & $\mathrm{H}$ & B & 22,27 \\
\hline 31 & Asteraceae & Chrysanthemum & myconis & $\mathrm{L}$. & 0 & 0 & 1 & $\mathrm{H}$ & $\mathrm{Y}$ & 27 \\
\hline 32 & Asteraceae & Helichrysum & bracteatum & (Vent.) Andrews & 0 & 0 & 1 & $\mathrm{H}$ & $\mathrm{R}$ & 14 \\
\hline 33 & Asteraceae & Leucanthemum & vulgare & Lam. & 0 & 0 & 1 & $\mathrm{H}$ & $\mathrm{W}$ & 7,18 \\
\hline 34 & Asteraceae & Senecio & bonariensis & Hook. \& Arn. & 1 & 1 & 0 & $\mathrm{H}$ & W & 18 \\
\hline 35 & Asteraceae & Senecio & confusus & Britten & 0 & 0 & 1 & $\mathrm{~V}$ & $\mathrm{R}$ & 20 \\
\hline 36 & Asteraceae & Senecio & icoglossus & DC. & 1 & 0 & 0 & $\mathrm{H}$ & $\mathrm{L}$ & $\begin{array}{l}18,22, \\
24,25\end{array}$ \\
\hline 37 & Asteraceae & Senecio & pulcher & Hook. \& Arn. & 1 & 0 & 0 & $\mathrm{H}$ & PI & 16 \\
\hline 38 & Asteraceae & Tithonia & diversifolia & (Helmsl.) A. Gray & 0 & 0 & 1 & S & $\mathrm{Y}$ & 7,20 \\
\hline 39 & Asteraceae & Trichocline & catharinensis & Cabrera & 1 & 1 & 0 & $\mathrm{H}$ & $\mathrm{Y}$ & 7,11 \\
\hline
\end{tabular}




\section{Continued}

\begin{tabular}{|c|c|c|c|c|c|c|c|c|c|c|}
\hline 40 & Asteraceae & Zinnia & elegans & Jacq. & 0 & 0 & 1 & $\mathrm{H}$ & W & 7,13 \\
\hline 41 & Asteraceae & Zinnia & peruviana & (L.) L. & 0 & 0 & 1 & $\mathrm{H}$ & $\mathrm{O}$ & 17 \\
\hline 42 & Balsaminaceae & Impatiens & walleriana & Hook. f. & 0 & 0 & 1 & $\mathrm{H}$ & $\mathrm{W}$ & $2,7,8$ \\
\hline 43 & Begoniaceae & Begonia & descoleana & $\begin{array}{l}\text { L.B. Sm. \& B.G. } \\
\text { Schub. }\end{array}$ & 1 & 0 & 0 & $\mathrm{H}$ & W & 26 \\
\hline 44 & Begoniaceae & Begonia & convolvulacea & (Klotzsch) A.DC. & 1 & 1 & 0 & $\mathrm{~V}$ & W & 28 \\
\hline 45 & Begoniaceae & Begonia & fischeri & Schrank & 1 & 0 & 0 & $\mathrm{H}$ & W & 27 \\
\hline 46 & Bignoniaceae & Amphilophium & crucigerum & (L.) L.G.Lohmann & 1 & 0 & 0 & $\mathrm{~V}$ & $\mathrm{~W}$ & 18 \\
\hline 47 & Bignoniaceae & Tanaecium & selloi & (Spreng.) Sandw. & 1 & 0 & 0 & $\mathrm{~V}$ & PI & 5,7 \\
\hline 48 & Bignoniaceae & Handroanthus & alba & (Cham.) Mattos & 1 & 0 & 0 & $\mathrm{~T}$ & $\mathrm{~W}$ & 3 \\
\hline 49 & Bignoniaceae & Handroanthus & ochraceus & (Cham.) Mattos & 1 & 0 & 0 & $\mathrm{~T}$ & $\mathrm{Y}$ & 12 \\
\hline 50 & Bignoniaceae & Jacaranda & puberula & Cham. & 1 & 1 & 0 & $\mathrm{~T}$ & $\mathrm{~L}$ & 5 \\
\hline 51 & Bignoniaceae & Pyrostegia & ignea & (Vell.) C. Presl & 0 & 0 & 1 & $\mathrm{~V}$ & $\mathrm{O}$ & 17 \\
\hline 52 & Bignoniaceae & Tabebuia & umbellata & (Sond.) Sandw. & 1 & 0 & 0 & $\mathrm{~T}$ & $\mathrm{Y}$ & 7 \\
\hline 53 & Boraginaceae & Echium & plantagineum & L. & 0 & 0 & 1 & $\mathrm{H}$ & B & 14 \\
\hline 54 & Brassicaceae & Alyssum & maritimum & (L.) Lam & 0 & 0 & 1 & $\mathrm{H}$ & W & 22 \\
\hline 55 & Bromeliaceae & Aechmea & cylindrata & Lindm. & 1 & 1 & 0 & $\mathrm{E}$ & PI & 16 \\
\hline 56 & Bromeliaceae & Vriesea & incurvata & Gaudich. & 1 & 1 & 0 & $\mathrm{H}$ & $\mathrm{R}$ & 20 \\
\hline 57 & Bromeliaceae & Vriesea & vagans & (L.B.Sm.) L.B.Sm. & 1 & 1 & 0 & $\mathrm{H}$ & $\mathrm{R}$ & 20 \\
\hline 58 & Cactaceae & Opuntia & monacantha & Haw. & 1 & 0 & 0 & $\mathrm{~S}$ & $\mathrm{Y}$ & 20 \\
\hline 59 & Cactaceae & Opuntia & ficus-indica & (L.) Mill. & 0 & 0 & 1 & $\mathrm{~S}$ & $\mathrm{Y}$ & 20 \\
\hline 60 & Cactaceae & Parodia & haselbergii & $\begin{array}{c}\text { (Haage ex Rümpler) } \\
\text { Brandt }\end{array}$ & 1 & 0 & 0 & $\mathrm{H}$ & $\mathrm{O}$ & 20,25 \\
\hline 61 & Cactaceae & Rhipsalis & elliptica & $\begin{array}{l}\text { G.Lindb. ex } \\
\text { K.Schum. }\end{array}$ & 1 & 1 & 0 & $\mathrm{H}$ & $\mathrm{W}$ & 28 \\
\hline 62 & Cactaceae & Napalea & cochenillifera & (L.) Salm-Dyck & 1 & 0 & 0 & $\mathrm{H}$ & $\mathrm{R}$ & 3 \\
\hline 63 & Cactaceae & Schlumbergera & truncata & (Haw.) Moran & 1 & 1 & 0 & $\mathrm{H}$ & W & 12 \\
\hline 64 & Campanulaceae & Hippobroma & longiflora & (Willd.) Presl. & 1 & 0 & 0 & $\mathrm{H}$ & W & 2,7 \\
\hline 65 & Campanulaceae & Lobelia & camporum & Pohl. & 1 & 0 & 0 & $\mathrm{H}$ & $\mathrm{W}$ & $10,11,20$ \\
\hline 66 & Campanulaceae & Wahlenbergia & linarioides & (Lam.) A. DC. & 1 & 0 & 0 & $\mathrm{H}$ & W & $\begin{array}{l}9,10 \\
11,20\end{array}$ \\
\hline 67 & Cannaceae & Canna & pedunculata & Sims & 1 & 1 & 0 & $\mathrm{H}$ & $\mathrm{R}$ & $\begin{array}{l}7,20 \\
25\end{array}$ \\
\hline 68 & Caprifoliaceae & Abelia & $\times$ grandiflora & & 0 & 0 & 1 & $\mathrm{~S}$ & PI & 20 \\
\hline 69 & Caryophyllaceae & Silene & pendula & Salz. ex. Willk. & 0 & 0 & 1 & $\mathrm{H}$ & PI & 27 \\
\hline 70 & Commelinaceae & Commelina & erecta & L. & 1 & 0 & 0 & $\mathrm{H}$ & B & 2,22 \\
\hline 71 & Commelinaceae & Tradescantia & fluminensis & Vell. & 1 & 0 & 0 & $\mathrm{H}$ & W & 7,27 \\
\hline 72 & Commelinaceae & Gibasis & schiedeana & (Kunth) D.R. Hunt & 0 & 0 & 1 & $\mathrm{~V}$ & $\mathrm{~W}$ & 22 \\
\hline 73 & Convolvulaceae & Evolvulus & glomeratus & Ness. \& Mart & 1 & 0 & 0 & $\mathrm{H}$ & B & $13,18,25$ \\
\hline 74 & Convolvulaceae & Ipomoea & alba & L. & 1 & 0 & 0 & $\mathrm{~V}$ & W & $\begin{array}{l}7,18 \\
25\end{array}$ \\
\hline 75 & Convolvulaceae & Ipomoea & batatoides & Benth & 1 & 0 & 0 & $\mathrm{~V}$ & PI & 17,19 \\
\hline 76 & Convolvulaceae & Ipomoea & carnea & Jacq. & 1 & 0 & 0 & $\mathrm{~V}$ & PI & 7,27 \\
\hline 77 & Convolvulaceae & Ipomoea & coccinea & L. & 0 & 0 & 1 & $\mathrm{~V}$ & $\mathrm{R}$ & 5,19 \\
\hline 78 & Crassulaceae & Echeveria & elegans & Rose & 0 & 0 & 1 & $\mathrm{H}$ & $\mathrm{R}$ & 20,25 \\
\hline 79 & Crassulaceae & Sedum & multiceps & Cosson et Durieu & 0 & 0 & 1 & $\mathrm{H}$ & $\mathrm{Y}$ & $\begin{array}{l}5,18 \\
22,25\end{array}$ \\
\hline 80 & Euphorbiaceae & Aleurites & fordii & Steud & 0 & 0 & 1 & $\mathrm{~T}$ & PI & 17,22 \\
\hline
\end{tabular}




\section{Continued}

\begin{tabular}{|c|c|c|c|c|c|c|c|c|c|c|}
\hline 81 & Euphorbiaceae & Euphorbia & pulcherrima & Willd. ex Klotzsch & 0 & 0 & 1 & $\mathrm{~S}$ & $\mathrm{R}$ & 26 \\
\hline 82 & Euphorbiaceae & Euphorbia & milii & Des. Moul & 0 & 0 & 1 & $\mathrm{~S}$ & $\mathrm{R}$ & 14 \\
\hline 83 & Euphorbiaceae & Jatropha & multifida & L. & 0 & 0 & 1 & $\mathrm{~S}$ & $\mathrm{R}$ & 26 \\
\hline 84 & Fabaceae & Acacia & decurrens & Willd & 0 & 0 & 1 & $\mathrm{~T}$ & $\mathrm{Y}$ & 17 \\
\hline 85 & Fabaceae & Acacia & mollissima & Willd. & 0 & 0 & 1 & $\mathrm{~T}$ & $\mathrm{Y}$ & 17 \\
\hline 86 & Fabaceae & Acacia & podalyriifolia & A. Cunn. ex G. Don & 0 & 0 & 1 & $\mathrm{~T}$ & $\mathrm{Y}$ & 17,18 \\
\hline 87 & Fabaceae & Bauhinia & forficata & Link & 1 & 0 & 0 & $\mathrm{~T}$ & $\mathrm{~W}$ & $17,18,20$ \\
\hline 88 & Fabaceae & Calliandra & brevipes & Beth. & 1 & 0 & 0 & $\mathrm{~S}$ & PI & 13 \\
\hline 89 & Fabaceae & Calliandra & dysantha & Benth & 1 & 0 & 0 & $\mathrm{~S}$ & $\mathrm{R}$ & 27 \\
\hline 90 & Fabaceae & Calliandra & foliolosa & Benth. & 1 & 0 & 0 & $\mathrm{~T}$ & $\mathrm{R}$ & 18 \\
\hline 91 & Fabaceae & Calliandra & longipes & Benth. & 1 & 0 & 0 & $\mathrm{H}$ & PI & 17 \\
\hline 92 & Fabaceae & Cassia & chamaechrista & Linn & 0 & 0 & 1 & $\mathrm{H}$ & $\mathrm{Y}$ & 17 \\
\hline 93 & Fabaceae & Dioclea & violacea & Mart. ex Benth. & 1 & 0 & 0 & $\mathrm{~V}$ & $\mathrm{P}$ & 16 \\
\hline 94 & Fabaceae & Erythrina & crista-galli & L. & 1 & 0 & 0 & $\mathrm{~T}$ & $\mathrm{R}$ & 7 \\
\hline 95 & Fabaceae & Erythrina & verna & Vell. & 1 & 1 & 0 & $\mathrm{~T}$ & $\mathrm{R}$ & $17,18,26$ \\
\hline 96 & Fabaceae & Lablab & purpureus & (L.) Sweet & 0 & 0 & 1 & $\mathrm{~V}$ & $\mathrm{P}$ & 17 \\
\hline 97 & Fabaceae & Libidibia & ferrea & $\begin{array}{l}\text { (Mart. ex Tul.) } \\
\text { L.P.Queiroz }\end{array}$ & 1 & 1 & 0 & $\mathrm{~T}$ & $\mathrm{Y}$ & 17 \\
\hline 98 & Fabaceae & Mimosa & per-dusenii & Burkart & 1 & 1 & 0 & SS & PI & $\begin{array}{l}7,19 \\
20,25\end{array}$ \\
\hline 99 & Fabaceae & Peltophorum & dubium & (Spreng.) Taub. & 1 & 0 & 0 & $\mathrm{~T}$ & $\mathrm{Y}$ & 28 \\
\hline 100 & Fabaceae & Senna & pendula & $\begin{array}{c}\text { (Humb. \& Bonpl. ex } \\
\text { Willd.) H.S. Irwin \& } \\
\text { Barneby }\end{array}$ & 1 & 0 & 0 & $\mathrm{~S}$ & $\mathrm{Y}$ & 1 \\
\hline 101 & Fabaceae & Stylosanthes & viscosa & SW & 1 & 0 & 0 & SS & $\mathrm{Y}$ & 3 \\
\hline 102 & Fabaceae & Trifolium & campestre & Sehr. & 0 & 0 & 1 & $\mathrm{H}$ & $\mathrm{Y}$ & 9 \\
\hline 103 & Fabaceae & Vicia & cracca & L. & 0 & 0 & 1 & $\mathrm{H}$ & B & 7 \\
\hline 104 & Fabaceae & Vicia & villosa & Roth & 0 & 0 & 1 & SS & B & 17 \\
\hline 105 & Fabaceae & Wisteria & sinensis & (Sims) Sweet & 0 & 0 & 1 & $\mathrm{~V}$ & $\mathrm{~L}$ & 12 \\
\hline 106 & Gesneriaceae & Seemannia & sylvatica & (Kunth) Hanst. & 1 & 1 & 0 & $\mathrm{H}$ & $\mathrm{O}$ & 7,24 \\
\hline 107 & Heliconiaceae & Heliconia & velloziana & Emygdio & 0 & 0 & 1 & $\mathrm{~S}$ & $\mathrm{R}$ & 14 \\
\hline 108 & Iridaceae & Neomarica & candida & (Hassl.) Sprague & 1 & 0 & 0 & $\mathrm{H}$ & $\mathrm{W}$ & 16 \\
\hline 109 & Lamiaceae & Clerodendrum & thomsoniae & Balf & 0 & 0 & 1 & $\mathrm{~V}$ & $\mathrm{~W}$ & 22 \\
\hline 110 & Lamiaceae & Coleus & blumei & Benth. & 0 & 0 & 1 & $\mathrm{H}$ & $\mathrm{B}$ & 25 \\
\hline 111 & Lamiaceae & Cunila & galioides & Benth. & 1 & 0 & 0 & $\mathrm{H}$ & $\mathrm{L}$ & $10,11,22$ \\
\hline 112 & Lamiaceae & Holmskioldia & sanguinea & Retz. & 0 & 0 & 1 & $\mathrm{H}$ & $\mathrm{R}$ & 22,27 \\
\hline 113 & Lamiaceae & Lavandula & dentata & L. & 0 & 0 & 1 & $\mathrm{H}$ & $\mathrm{L}$ & 27 \\
\hline 114 & Lamiaceae & Mentha & pulegium & L. & 0 & 0 & 1 & $\mathrm{H}$ & $\mathrm{L}$ & 2,8 \\
\hline 115 & Lamiaceae & Plectranthus & amboinicus & (Lour.) Spreng & 0 & 0 & 1 & $\mathrm{H}$ & $\mathrm{L}$ & 7 \\
\hline 116 & Lamiaceae & Solenostemon & scutellarioides & (L.) Codd. & 0 & 0 & 1 & $\mathrm{H}$ & B & 1 \\
\hline 117 & Liliaceae & Agapanthus & africanus & (L.) Hoffsgg. & 0 & 0 & 1 & $\mathrm{H}$ & W & 7 \\
\hline 118 & Liliaceae & Lilium & regale & E. H. Wilson & 0 & 0 & 1 & $\mathrm{H}$ & $\mathrm{W}$ & 7,24 \\
\hline 119 & Linderniaceae & Torenia & fournieri & Linden ex E. Fourn. & 0 & 0 & 1 & $\mathrm{H}$ & B & 18,22 \\
\hline 120 & Malvaceae & Abutilon & megapotamicum & $\begin{array}{l}\text { (Spreng.) A.St.-Hil. } \\
\text { \& Naudin }\end{array}$ & 1 & 0 & 0 & $\mathrm{~S}$ & $\mathrm{R}$ & 9 \\
\hline 121 & Malvaceae & Ceiba & speciosa & $\begin{array}{l}\text { (A. St.-Hil.) } \\
\text { Ravenna }\end{array}$ & 1 & 1 & 0 & $\mathrm{~T}$ & PI & 17,25 \\
\hline
\end{tabular}




\section{Continued}

\begin{tabular}{|c|c|c|c|c|c|c|c|c|c|c|}
\hline 122 & Malvaceae & Dombeya & natalensis & Sond & 0 & 0 & 1 & $\mathrm{~T}$ & $\mathrm{~W}$ & 17,25 \\
\hline 123 & Malvaceae & Dombeya & wallichii & (Lindl.) K. Schum. & 0 & 0 & 1 & $\mathrm{~T}$ & PI & 9 \\
\hline 124 & Malvaceae & Hibiscus & sabdariffa & $\mathrm{L}$. & 0 & 0 & 1 & $\mathrm{~S}$ & $\mathrm{Y}$ & 7 \\
\hline 125 & Malvaceae & Hibiscus & rosa-sinensis & L. & 0 & 0 & 1 & $\mathrm{~S}$ & W & 20,25 \\
\hline 126 & Marantaceae & Stromanthe & sanguinea & Sond. & 0 & 0 & 1 & $\mathrm{~S}$ & $\mathrm{R}$ & 20,27 \\
\hline 127 & Melastomataceae & Tibouchina & heteromalla & (D.Don) Cogn. & 1 & 1 & 0 & $\mathrm{~S}$ & B & 7 \\
\hline 128 & Melastomataceae & Tibouchina & granulosa & (Desr.) Cogn. & 1 & 1 & 0 & $\mathrm{~T}$ & $\mathrm{P}$ & $\begin{array}{l}7,19, \\
20,25\end{array}$ \\
\hline 129 & Melastomataceae & Tibouchina & moricandiana & Baill. & 1 & 0 & 0 & $\mathrm{~S}$ & B & $\begin{array}{c}7,18, \\
21\end{array}$ \\
\hline 130 & Melastomataceae & Tibouchina & pilosa & Cogn. & 1 & 1 & 0 & $\mathrm{~T}$ & PI & $\begin{array}{c}8,18, \\
20\end{array}$ \\
\hline 131 & Myrsinaceae & Ardisia & crenata & Sims. & 0 & 0 & 1 & $\mathrm{~S}$ & $\mathrm{~W}$ & 7 \\
\hline 132 & Myrtaceae & Acca & sellowiana & (O.Berg) Burret & 1 & 0 & 0 & $\mathrm{~T}$ & W & 28 \\
\hline 133 & Myrtaceae & Callistemon & lanceolatus & Sweet & 0 & 0 & 1 & $\mathrm{~S}$ & $\mathrm{R}$ & 3 \\
\hline 134 & Nyctaginaceae & Bougainvillea & glabra & Choisy & 0 & 0 & 1 & $\mathrm{~S}$ & $\mathrm{Y}$ & 13,22 \\
\hline 135 & Nymphaeaceae & Nymphaea & caerulea & Savigny & 0 & 0 & 1 & $\mathrm{H}$ & $\mathrm{B}$ & 12 \\
\hline 136 & Oleaceae & Jasminum & sambac & (L.) Aiton & 0 & 0 & 1 & $\mathrm{~S}$ & W & 14 \\
\hline 137 & Oleaceae & Ligustrum & japonicum & Thunb. & 0 & 0 & 1 & $\mathrm{~T}$ & W & 17 \\
\hline 138 & Papaveraceae & Eschscholzia & californica & Cham. & 0 & 0 & 1 & $\mathrm{H}$ & $\mathrm{O}$ & 25 \\
\hline 139 & Papaveraceae & Papaver & somniferum & L. & 0 & 0 & 1 & $\mathrm{H}$ & W & 19 \\
\hline 140 & Passifloraceae & Passiflora & urubiciensis & Cervi. & 1 & 1 & 0 & V & $\mathrm{W}$ & 19,20 \\
\hline 141 & Passifloraceae & Passiflora & alata & Curtis & 1 & 1 & 0 & $\mathrm{~V}$ & $\mathrm{P}$ & $\begin{array}{c}7,13, \\
20\end{array}$ \\
\hline 142 & Passifloraceae & Passiflora & caerulea & L. & 1 & 0 & 0 & $\mathrm{~V}$ & W & $\begin{array}{c}9,19, \\
25\end{array}$ \\
\hline 143 & Passifloraceae & Passiflora & edulis & Sims & 1 & 0 & 0 & $\mathrm{~V}$ & W & 2,13 \\
\hline 144 & Plantaginaceae & Antirrhinum & majus & L. & 0 & 0 & 1 & $\mathrm{H}$ & W & 16 \\
\hline 145 & Plumbaginaceae & Plumbago & capensis & Thumb. & 0 & 0 & 1 & $\mathrm{~S}$ & $\mathrm{~B}$ & 7,22 \\
\hline 146 & Polemoniaceae & Phlox & drummondii & Hook. & 0 & 0 & 1 & $\mathrm{H}$ & $\mathrm{W}$ & 7 \\
\hline 147 & Polygalaceae & Monnina & cuneata & A. St.- Hil & 1 & 0 & 0 & $\mathrm{H}$ & $\mathrm{L}$ & 10,11 \\
\hline 148 & Polygonaceae & Antigonon & leptopus & Hook. \& Arn. & 0 & 0 & 1 & $\mathrm{~V}$ & PI & 17,22 \\
\hline 149 & Polygonaceae & Polygonum & capitatum & $\begin{array}{l}\text { Buch.-Ham. ex } \\
\text { D.Don }\end{array}$ & 0 & 0 & 1 & $\mathrm{H}$ & PI & $\begin{array}{c}7,18, \\
24\end{array}$ \\
\hline 150 & Portulacaceae & Portulaca & grandiflora & Hook. & 1 & 0 & 0 & $\mathrm{H}$ & PI & 18,25 \\
\hline 151 & Primulaceae & Anagallis & arvensis & L. & 1 & 0 & 0 & $\mathrm{H}$ & $\mathrm{O}$ & 25,27 \\
\hline 152 & Primulaceae & Primula & malacoides & Franch. & 0 & 0 & 1 & $\mathrm{H}$ & PI & 27 \\
\hline 153 & Proteaceae & Grevillea & banksii & R. Br. & 0 & 0 & 1 & $\mathrm{~T}$ & $\mathrm{R}$ & 17,22 \\
\hline 154 & Proteaceae & Grevillea & thelemanniana & Hueg. & 0 & 0 & 1 & $\mathrm{~S}$ & $\mathrm{R}$ & 17,22 \\
\hline 155 & Rhamnaceae & Colletia & exserta & Klotzsch ex Reissek & 1 & 0 & 0 & $\mathrm{~S}$ & W & 28 \\
\hline 156 & Rosaceae & Fragaria & vesca & $\mathrm{L}$. & 0 & 0 & 1 & $\mathrm{H}$ & $\mathrm{W}$ & 7,22 \\
\hline 157 & Rosaceae & Malus & pumila & Mill. & 0 & 0 & 1 & $\mathrm{~T}$ & $\mathrm{~W}$ & 22 \\
\hline 158 & Rosaceae & Prunus & amygdalus & Stokes. & 0 & 0 & 1 & $\mathrm{~T}$ & W & 18 \\
\hline 159 & Rosaceae & Prunus & armeniaca & L. & 0 & 0 & 1 & $\mathrm{~T}$ & $\mathrm{~W}$ & 17 \\
\hline 160 & Rosaceae & Prunus & domestica & L. & 0 & 0 & 1 & $\mathrm{~T}$ & W & $\begin{array}{c}9,17 \\
25\end{array}$ \\
\hline 161 & Rosaceae & Prunus & serrulata & Lindl. & 0 & 0 & 1 & $\mathrm{~T}$ & $\mathrm{~W}$ & 17 \\
\hline 162 & Rosaceae & Rosa & wichuraiana & Crép. & 0 & 0 & 1 & $\mathrm{~V}$ & W & 21 \\
\hline
\end{tabular}




\section{Continued}

\begin{tabular}{|c|c|c|c|c|c|c|c|c|c|c|}
\hline 163 & Rosaceae & Rosa & chinensis & Jacq. & 0 & 0 & 1 & $\mathrm{H}$ & PI & 7 \\
\hline 164 & Rosaceae & Rosa & gallica & L. & 0 & 0 & 1 & $\mathrm{~S}$ & PI & $17,18,19,23$ \\
\hline 165 & Rosaceae & Rubus & spectabilis & Pursh & 0 & 0 & 1 & $\mathrm{~S}$ & $\mathrm{P}$ & 23 \\
\hline 166 & Rubiaceae & Pentas & lanceolata & (Forssk.) Deflers & 0 & 0 & 1 & $\mathrm{H}$ & PI & 26 \\
\hline 167 & Rubiaceae & Coutarea & hexandra & K. Schum & 1 & 0 & 0 & $\mathrm{~T}$ & $\mathrm{~W}$ & 17 \\
\hline 168 & Rutaceae & Murraia & paniculata & Jack. & 0 & 0 & 1 & $\mathrm{~T}$ & $\mathrm{~W}$ & 17 \\
\hline 169 & Saxifragaceae & Escallonia & bifida & DC. & 1 & 0 & 0 & $\mathrm{~S}$ & $\mathrm{~W}$ & 17,22 \\
\hline 170 & Scrophulariaceae & Veronica & persica & Poir. & 0 & 0 & 1 & $\mathrm{H}$ & B & 7 \\
\hline 171 & Scrophulariaceae & Buddleja & stachyoides & Cham. \& Schltdl. & 1 & 0 & 0 & $\mathrm{H}$ & $\mathrm{Y}$ & 3 \\
\hline 172 & Solanaceae & Browallia & americana & L. & 1 & 0 & 0 & $\mathrm{~S}$ & $\mathrm{~L}$ & 12 \\
\hline 173 & Solanaceae & Brunfelsia & uniflora & (Pohl) D.Don & 1 & 0 & 0 & $\mathrm{~S}$ & $\mathrm{~W}$ & 14 \\
\hline 174 & Solanaceae & Calibrachoa & dusenii & $\begin{array}{l}\text { (R.E.Fr.) Stehmann } \\
\text { \& Semir }\end{array}$ & 1 & 1 & 0 & SS & $\mathrm{W}$ & 16 \\
\hline 175 & Solanaceae & Cestrum & corymbosum & Schltdl. & 1 & 1 & 0 & $\mathrm{~S}$ & $\mathrm{O}$ & 28 \\
\hline 176 & Solanaceae & Datura & metel & L. & 0 & 0 & 1 & $\mathrm{~S}$ & $\mathrm{~W}$ & 2,25 \\
\hline 177 & Solanaceae & Datura & suaveolens & $\begin{array}{l}\text { Humb. \& Bonpl. ex } \\
\text { Willd. }\end{array}$ & 0 & 0 & 1 & $\mathrm{~S}$ & $\mathrm{~W}$ & 2 \\
\hline 178 & Solanaceae & Nicandra & physalodes & (L.) Gaertn. & 0 & 0 & 1 & $\mathrm{~S}$ & $\mathrm{~L}$ & 19, 20 \\
\hline 179 & Solanaceae & Nicotiana & langsdorffii & Schrank & 1 & 0 & 0 & $\mathrm{H}$ & G & 5,19 \\
\hline 180 & Solanaceae & Petunia & bonjardinensis & T. Ando \&Hashim. & 1 & 1 & 0 & $\mathrm{H}$ & $\mathrm{P}$ & 18 \\
\hline 181 & Solanaceae & Petunia & scheideana & L.B.Sm. \& Downs & 1 & 0 & 0 & $\mathrm{H}$ & $\mathrm{P}$ & 3 \\
\hline 182 & Solanaceae & Solanum & $\begin{array}{l}\text { sanctae- } \\
\text { catharinae }\end{array}$ & Dunal & 1 & 0 & 0 & $\mathrm{~S}$ & $\mathrm{~W}$ & 18 \\
\hline 183 & Strelitziaceae & Strelitzia & reginae & Aiton & 0 & 0 & 1 & $\mathrm{H}$ & $\mathrm{O}$ & 13 \\
\hline 184 & Tamaricaceae & Tamarix & gallica & L. & 0 & 0 & 1 & $\mathrm{~T}$ & $\mathrm{~W}$ & $17,19,20,22$ \\
\hline 185 & Theaceae & Camellia & japonica & $\mathrm{L}$. & 0 & 0 & 1 & $\mathrm{~S}$ & $\mathrm{~W}$ & $7,17,25$ \\
\hline 186 & Tropaeolaceae & Tropaeolum & majus & L. & 0 & 0 & 1 & $\mathrm{H}$ & $\mathrm{Y}$ & $19,20,22$ \\
\hline 187 & Turneraceae & Turnera & ulmifolia & L. & 0 & 0 & 1 & $\mathrm{H}$ & $\mathrm{Y}$ & 12 \\
\hline 188 & Verbenaceae & Aloysia & virgata & Juss. & 1 & 0 & 0 & $\mathrm{~S}$ & $\mathrm{~W}$ & $\begin{array}{c}17,18,19 \\
20,21,23 \\
24,25\end{array}$ \\
\hline 189 & Verbenaceae & Duranta & erecta & $\mathrm{L}$. & 1 & 0 & 0 & $\mathrm{H}$ & $\mathrm{P}$ & $7,13,15$ \\
\hline 190 & Verbenaceae & Lantana & camara & L. & 1 & 0 & 0 & $\mathrm{~S}$ & $\mathrm{Y}$ & $\begin{array}{l}2,5,7,8,13, \\
17,18,20,23\end{array}$ \\
\hline 191 & Verbenaceae & Verbena & bonariensis & $\mathrm{L}$ & 1 & 0 & 0 & $\mathrm{H}$ & $\mathrm{L}$ & 14 \\
\hline 192 & Verbenaceae & Verbena & hirta & Dusen & 1 & 0 & 0 & $\mathrm{H}$ & $\mathrm{L}$ & $11,20,23$ \\
\hline 193 & Verbenaceae & Vitex & megapotamica & (Spreng.) Moldenke & 1 & 0 & 0 & $\mathrm{~T}$ & $\mathrm{~L}$ & 4,5 \\
\hline 194 & Verbenaceae & Vitex & sellowiana & Cham. & 1 & 1 & 0 & $\mathrm{H}$ & $\mathrm{W}$ & 17,19 \\
\hline 195 & Xanthorrhoeaceae & Hemerocallis & lilioasphodelus & L. & 0 & 0 & 1 & $\mathrm{H}$ & $\mathrm{Y}$ & 27 \\
\hline 196 & Xanthorrhoeaceae & Aloe & arborescens & Mill. & 0 & 0 & 1 & $\mathrm{H}$ & $\mathrm{O}$ & 19 \\
\hline 197 & Xanthorrhoeaceae & Aloe & ferox & Mill. & 0 & 0 & 1 & $\mathrm{H}$ & $\mathrm{R}$ & 26 \\
\hline 198 & Xanthorrhoeaceae & Aloe & vera & (L.) Burm. f. & 0 & 0 & 1 & $\mathrm{H}$ & $\mathrm{Y}$ & 24 \\
\hline 199 & Xanthorrhoeaceae & Bulbine & frutescens & (L.) Willd. & 0 & 0 & 1 & $\mathrm{H}$ & $\mathrm{Y}$ & 27 \\
\hline 200 & Zingiberaceae & Alpinia & zerumbet & $\begin{array}{l}\text { (Pers.) B.L.Burtt \& } \\
\text { R.M.Sm. }\end{array}$ & 0 & 0 & 1 & $\mathrm{H}$ & $\mathrm{W}$ & 19 \\
\hline 201 & Zingiberaceae & Hedychium & coronarium & Koenig & 0 & 0 & 1 & $\mathrm{H}$ & $\mathrm{W}$ & $\begin{array}{c}7,8,18,19 \\
21,26\end{array}$ \\
\hline
\end{tabular}

Legend: B = blue; $\mathrm{BU}$ = burgundy; Col. = color of the flower/inflorescence; End. = endemic of Brazil; Exot./introd. = exotic/introduced; $\mathrm{G}=$ green; $\mathrm{H}$ = herb; $\mathrm{Ha}=$ habitus; $\mathrm{L}=$ lilac; $\mathrm{N}=$ native; $\mathrm{O}$ = orange; $\mathrm{P}=$ purple; $\mathrm{PI}=$ pink; $\mathrm{R}=$ red; $\mathrm{S}=$ shrub; $\mathrm{SS}=$ subshrub; $\mathrm{T}=$ tree; $\mathrm{V}=$ vine; $\mathrm{W}=$ white; $\mathrm{Y}=$ yellow; $1=$ this feature; $0=$ not this feature. 


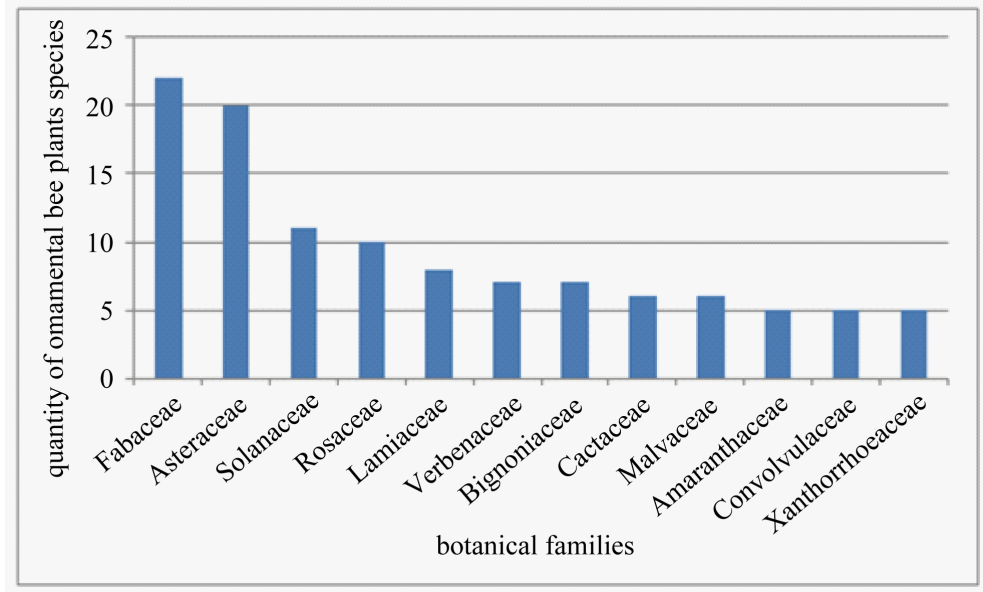

Figure 2. Quantity of ornamental bee plants species per botanical families, in Santa Catarina State, southern Brazil.

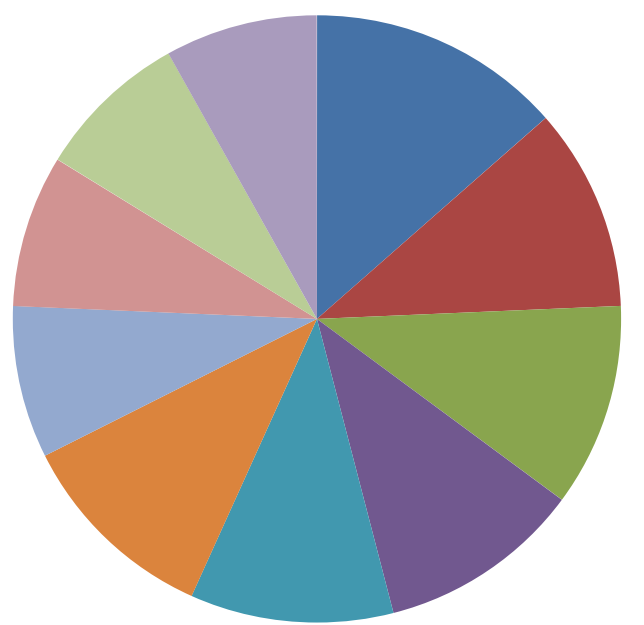

$$
\begin{aligned}
& \text { - Ipomoea } \\
& \text { - Calliandra } \\
& \text { - Passiflora } \\
& \text { - Prunus } \\
& \text { - Senecio } \\
& \text { - Tibouchina } \\
& \text { - Acacia } \\
& \text { - Aloe } \\
& \text { - Begonia } \\
& \text { - Rosa }
\end{aligned}
$$

Figure 3. Quantity of ornamental bee plants species per botanical genus, in Santa Catarina State, southern Brazil.
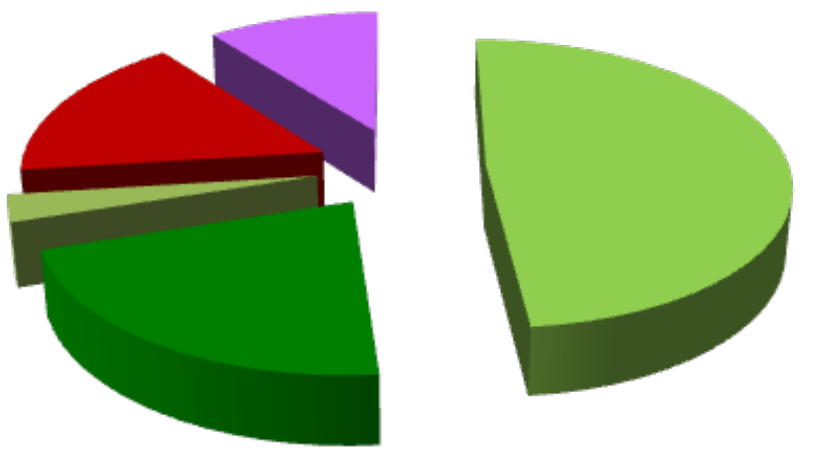

$$
\begin{aligned}
& \text { - Herb } \\
& \text {-Shrub } \\
& \text { - Subshrub } \\
& \text { - Tree } \\
& \text { - Vine }
\end{aligned}
$$

Figure 4. Proportion of habitus of the ornamental bee plant species, in Santa Catarina State, southern Brazil.

Regarding the origin of bee ornamental plants, 109 are exotic/introduced (54.2\%) and 92 are native (45.8\%), of which 26 (12.9\%) are endemic. The most cited ornamental bee plant species are: Lantana camara L. (native) (Verbenaceae) (9 quotes), Aloysia virgata (Ruiz \& Pav.) Juss. (native) (Verbenaceae) (8), Hedychium coronarium 


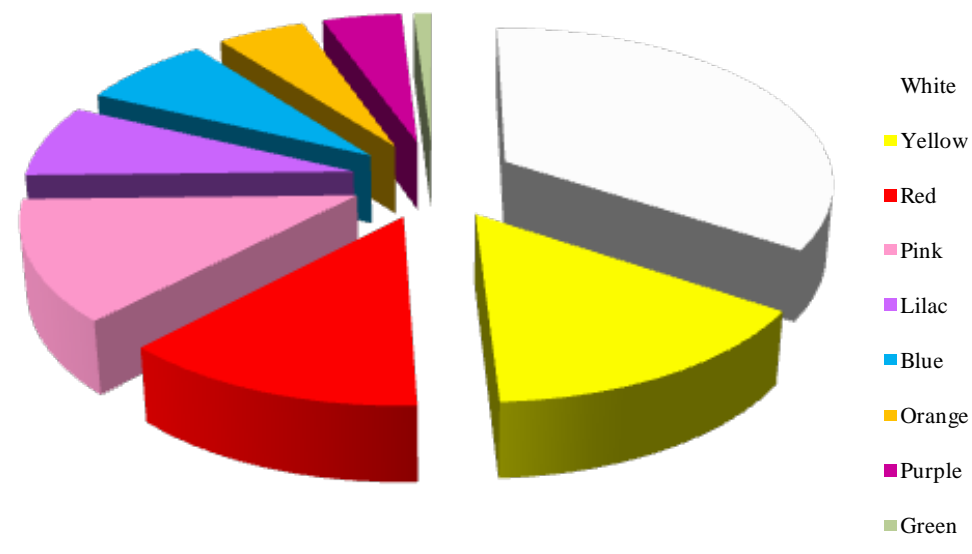

Figure 5. Proportion of colours in the ornamental bee plant species, in Santa Catarina State, southern Brazil.

J. Koenig (naturalized) (Zingiberaceae) (6) and Brachyscome multifida DC (endemic) (Asteraceae) (5). According to [20], native bees use a wide variety of ornamentals and their abundance depend on the plant type. Exotic taxa found corroborate studies conducted in Brazil and other countries about the presence of introduced/invasive/naturalized plant species, which insert in the environments where they penetrate and with which native bee species interact [32]. Despite the fact that most gardens use a high percentage of nonnative plants (instead of the native plants preferred by native bees), they are nonetheless visited by native bees [28]. As stated by [33], a garden plant need not be native to attract and feed native bees.

The results show a large botanical diversity, demonstrating the varied possibilities of utilization of ornamental plants as bee pasture species in urban beekeeping (Figure 6). The botanical families that had more ornamental bee plant species are those that encompass more taxa and so their ratio reflects their taxonomic richness too [34]. On the other hand, the fact that there is a proportion of endemic ornamental bee species adds an element of potential economic importance to the issue in focus since the production of ornamental plants is a booming economic activity in Brazil [35]. The prevalence of colors of flowers/inflorescences preferred by bees is reported by other authors [14] and should be considered when implementing urban gardening and landscaping for pollinators.

\section{Conclusions}

Even though urbanization has a negative effect on insect fauna, wild bees are found in urban environments [36]. Urban bees are those that lived in an area prior to urbanization and are able to adapt to anthropogenic alterations to the environment besides the exotic species that have become naturalized therein [20]. On the other hand, in urban environments, botanical species with different flowering periods are usually used in gardening, which favors the ornamentation factor [37] and, consequently, the supply of resources is maintained throughout the seasons. Urban plants also are usually intensively managed: watering, pruning and replanting produces floral resources that are more consistently available to pollinators, even in times of drought [20]. In urban environments, temperature is a little higher than outside city and pesticides are of restricted use in there [4].

Ornamental plants are not often thought as bee plants because they do not always offer conspicuous pollen or nectar resources [38]. Moreover, frequent attributes of ornamental plants such as double petals, no stamens, no nectar guides, strong scents, among others, drive off bees [39]. However, many of them are suitable for bees that visit them prominently. Nowadays there is a tendency of consumers for ornamental plants with sustainable attributes [40]. So, studies made up about the relations between flowers and native bees, summarized into lists of ornamental bee plants, can allow gardens to be planned to attract them. The potential consequences of pollinator decline on the preservation of biodiversity and stability of food crop yields should guide the policies of pollinator conservation [41].

\section{Acknowledgements}

To the experts Karin Esemann de Quadros and Cynthia Hering Rinnert of the Herbarium Joinvillea of the University of the Region of Joinville, and to the experts Osmar dos Santos Ribas, Juarez Cordeiro and Eraldo Barboza 

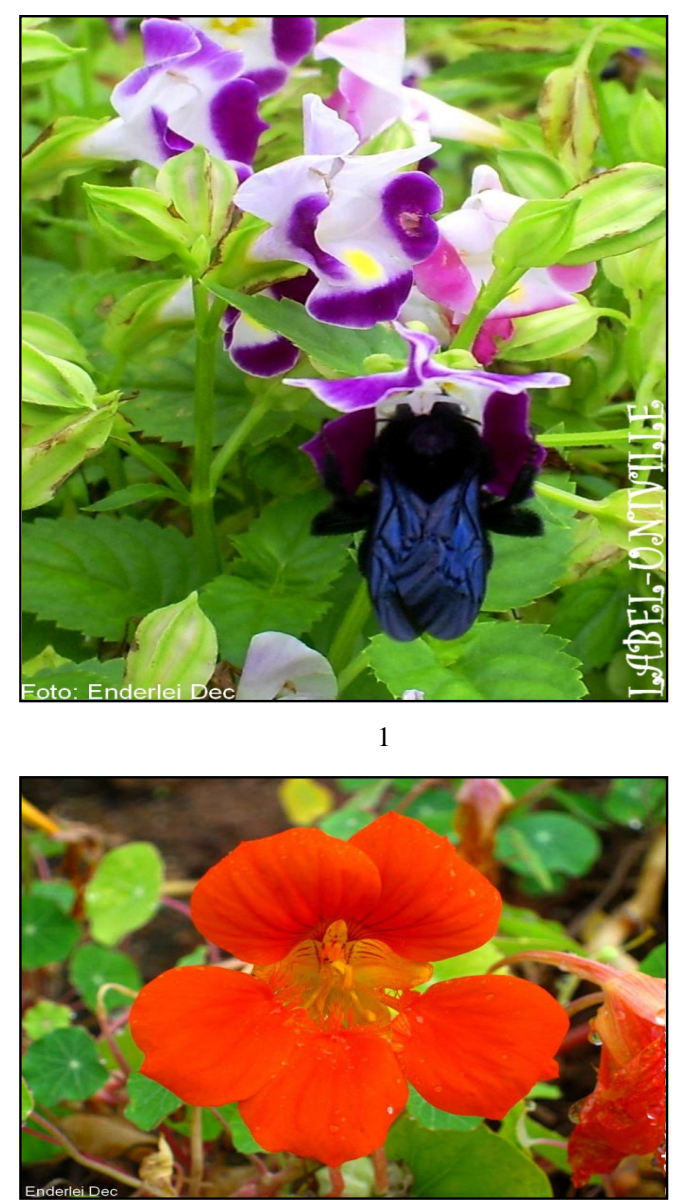

3

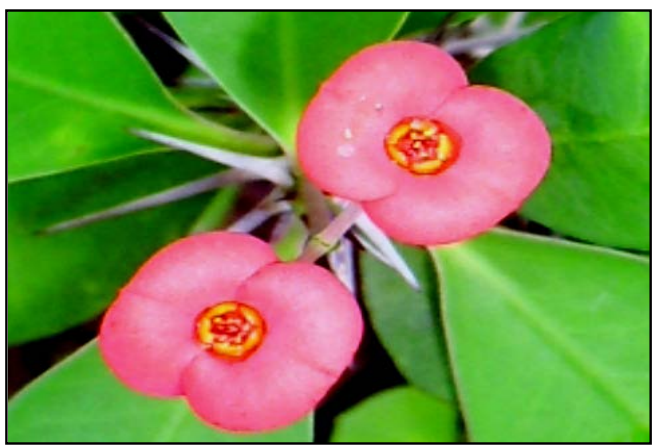

5

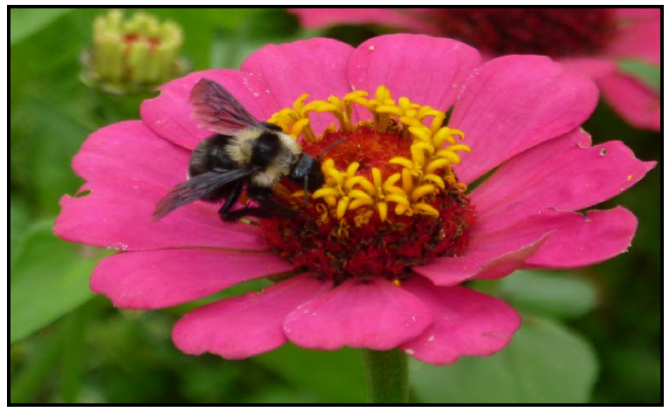

7
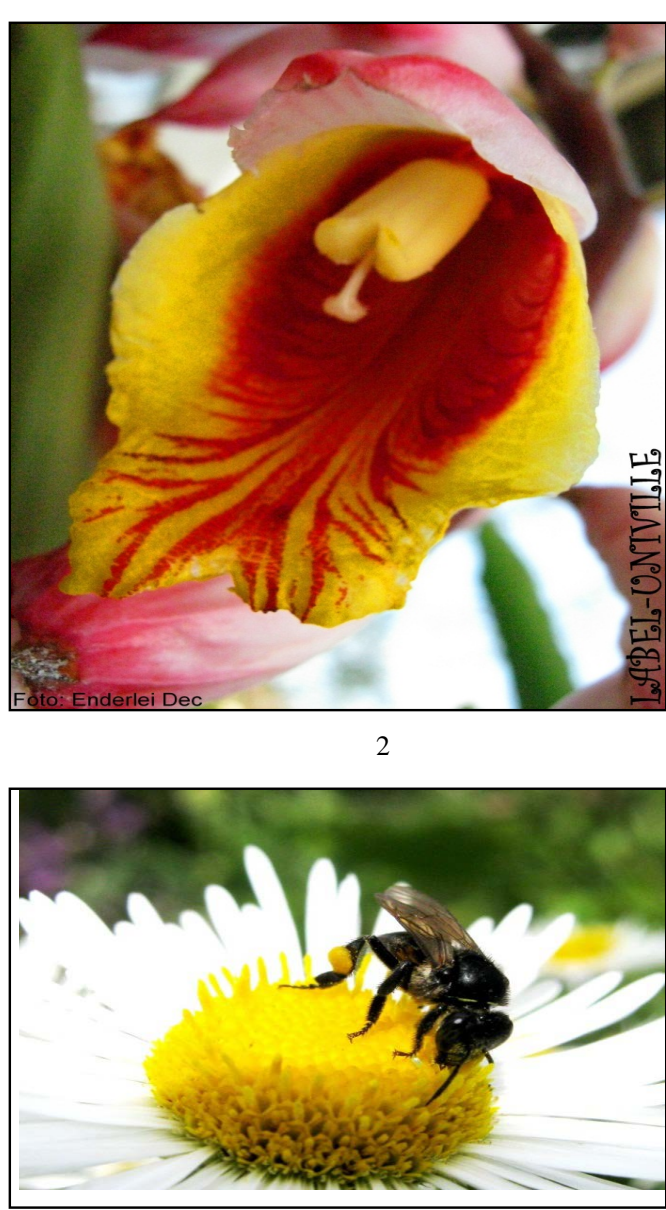

4

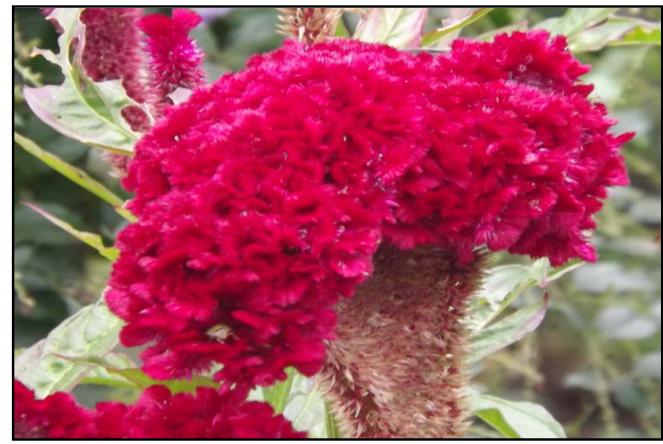

6

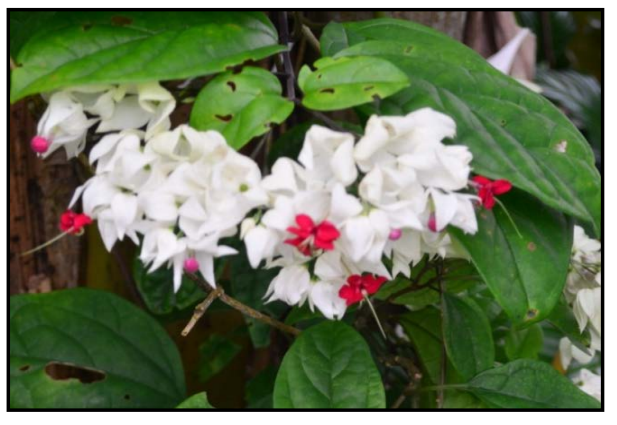

8 


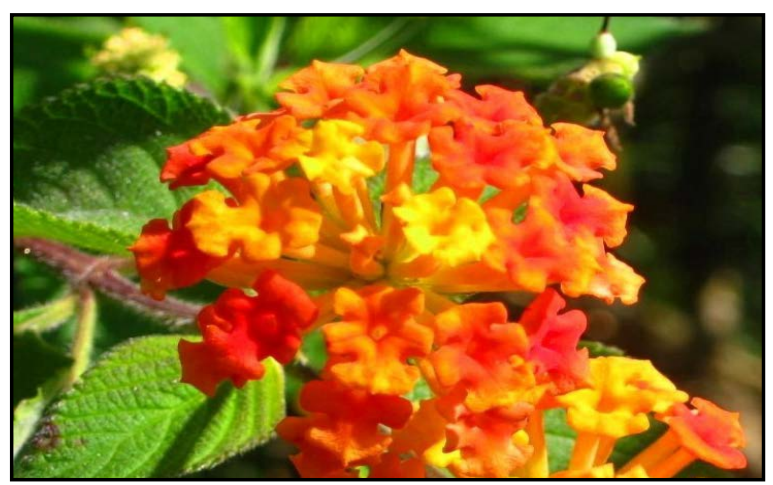

9

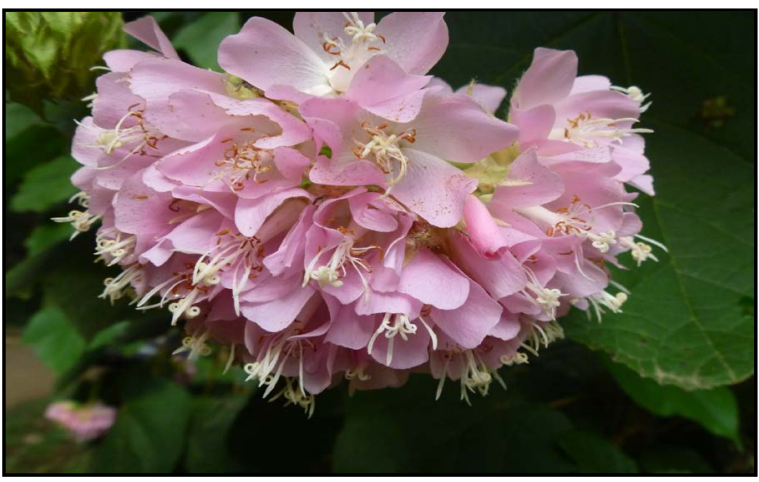

11

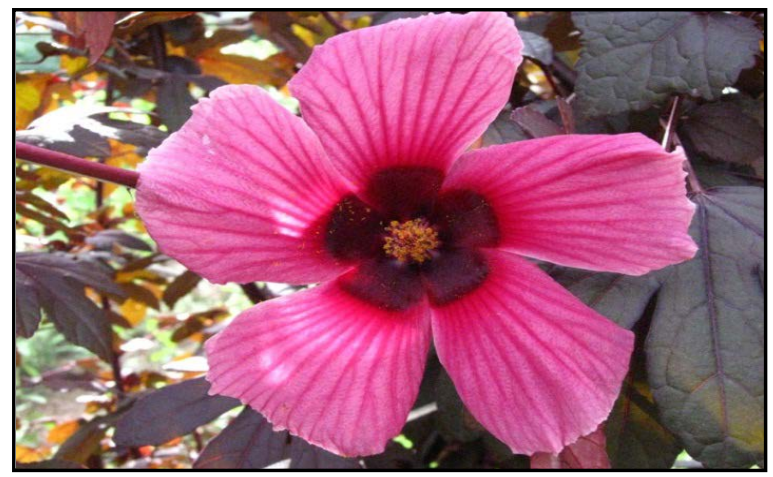

13

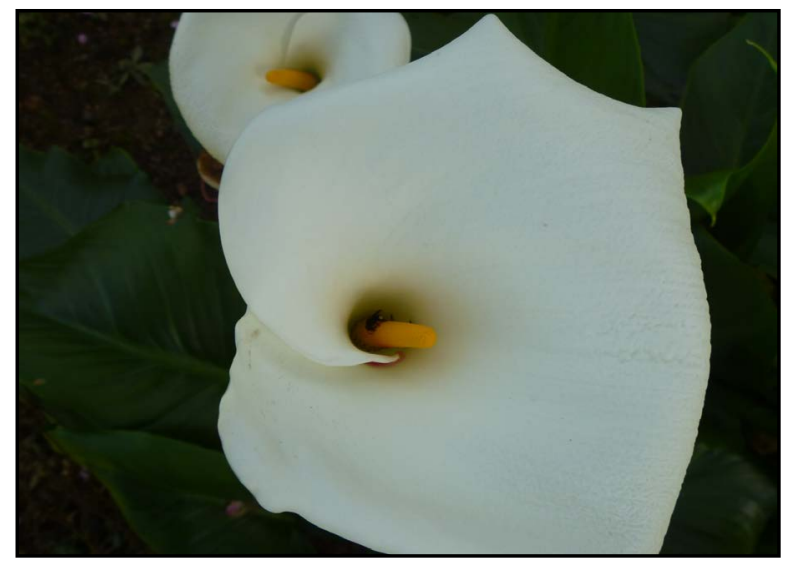

15

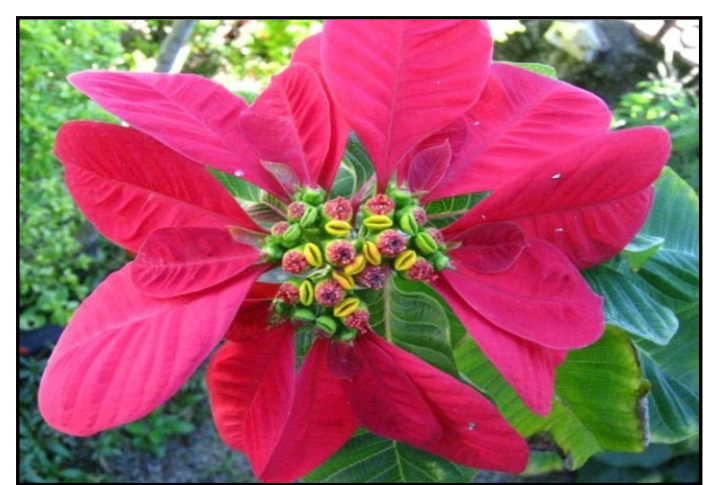

10

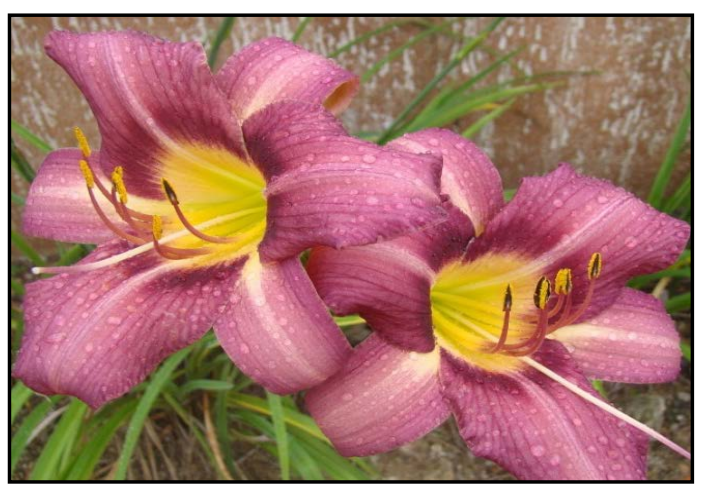

12

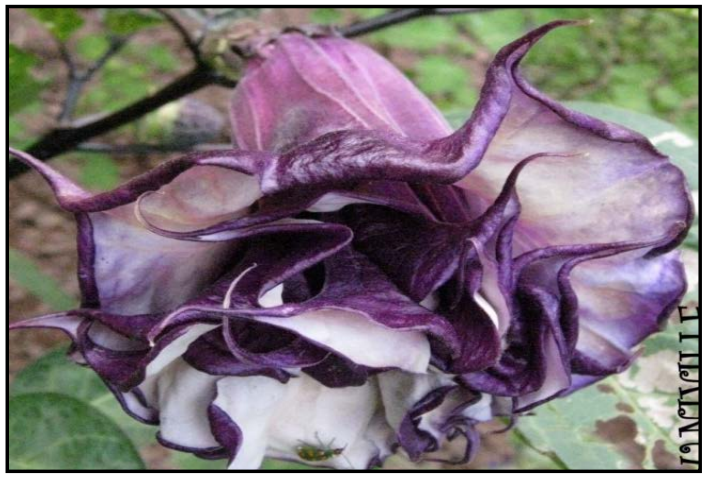

14

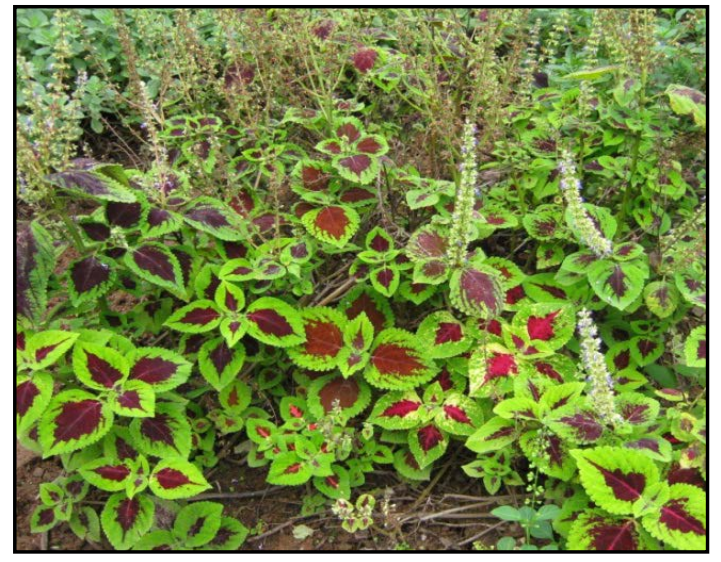

16 


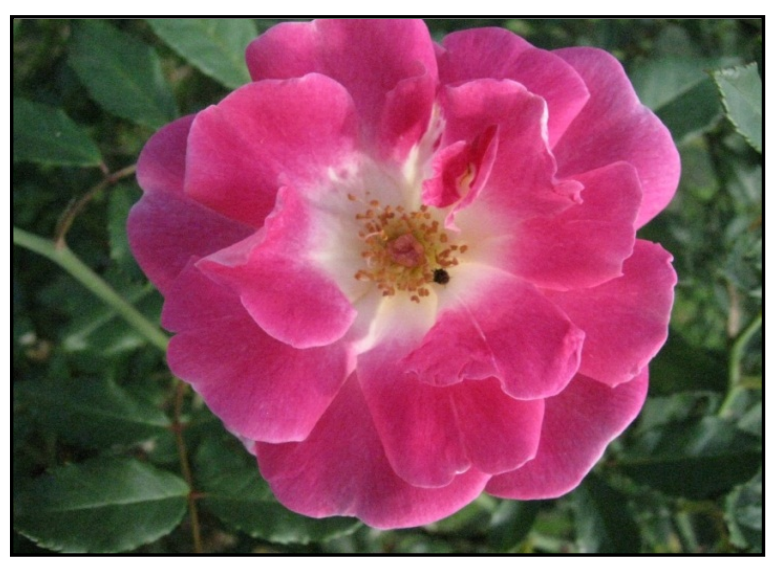

17

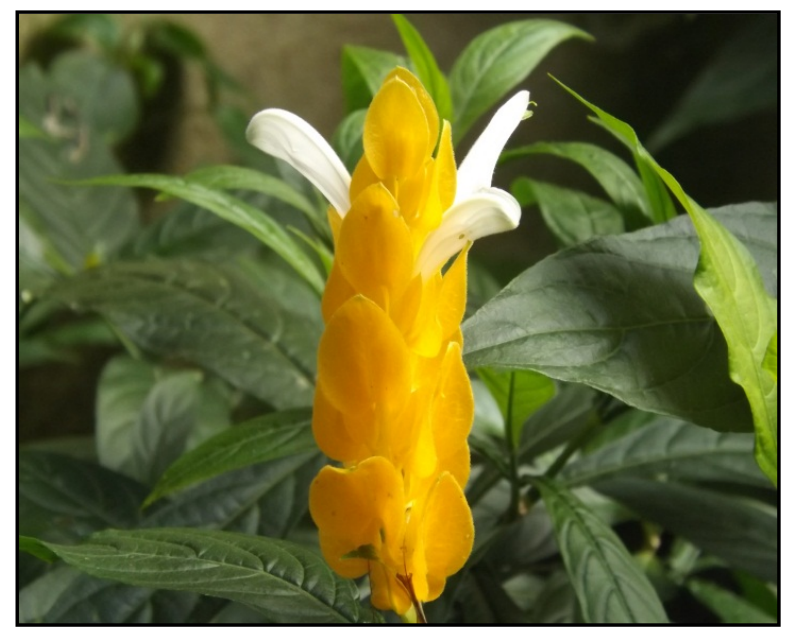

19

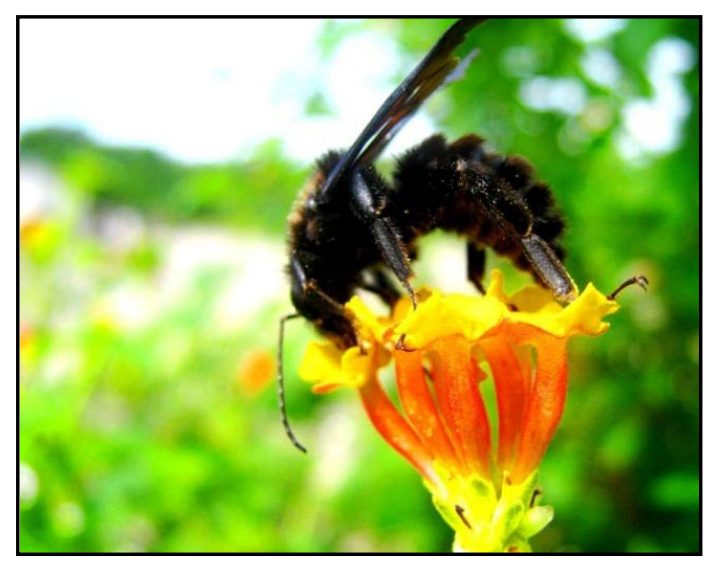

18

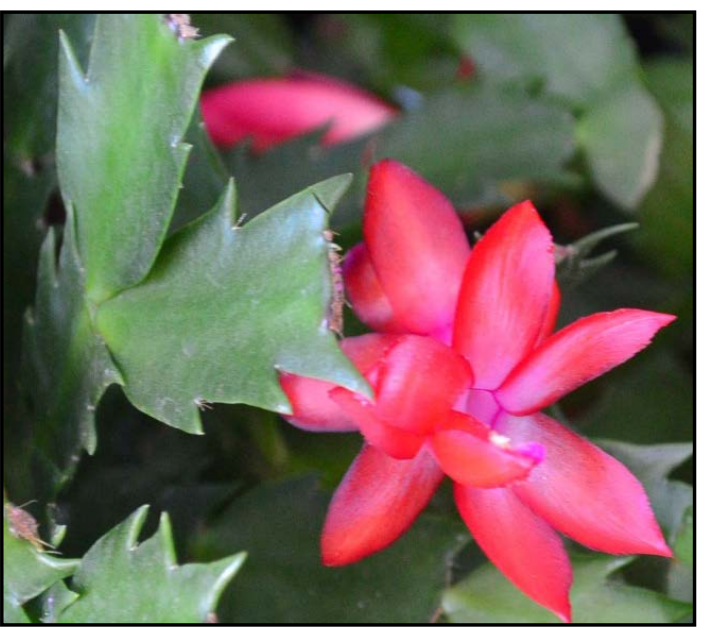

20

Figure 6. 1-Torrenia fournieri; 2-Alpinia zerumbet; 3-Tropaeolum majus; 4-Leptostelma maxima; 5-Euphorbia milii; 6Celosia cristata; 7-Zinnia elegans; 8-Clerodendrum thomsoniae; 9-Lantana camara; 10-Euphorbia pulcherrima; 11-Dombeya wallichii; 12-Hemerocallis flava; 13-Hibiscus sabdariffa; 14- Datura metel; 15-Zanthedeschia aethiopica; 16-Coleus blumei; 17-Rosa chinensis; 18-Cestrum corymbosum; 19-Justicia brandegeeana; 20-Schlumbergera truncata.

of the Botanical Museum of the City of Curitiba, for their assistance in botanical identification. To all those who contributed to this work.

\section{References}

[1] Gifford, C. (2011) Colony Collapse Disorder: The Vanishing Honeybee (Apis mellifera). Ph.D. Thesis, Colorado University, Boulder.

[2] Imperatriz-Fonseca, V.L., Canhos, D.A.L., Alves, D.A. and Saraiva, A.M. (2012) Pollinators and Pollination-A Global Issue. In: Imperatriz-Fonseca, V.L., Canhos, D.A.L., Alves, D.A. and Saraiva, A.M., Eds., Pollinators in Brazil-Contribution and Prospects for Biodiversity, Sustainable Use, Conservation and Environmental Services, EDUSP, São Paulo, 23-45.

[3] Frankie, G.W. and Ehler, L.E. (1978) Ecology of Insects in Urban Environments. Annual Review of Entomology, 23, 367-387. http://dx.doi.org/10.1146/annurev.en.23.010178.002055

[4] Fortel, L., Henry, M., Guilbaud, L., Guirao, A.L., Kuhlmann, M., Mouret, H., Rollin, O. and Vaissière, B.E. (2014) Decreasing Abundance, Increasing Diversity and Changing Structure of the Wild Bee Community (Hymenoptera: Anthophila) along an Urbanization Gradient. PLoS ONE, 9, e104679. http://dx.doi.org/10.1371/journal.pone.0104679

[5] Kevan, P.G. (1999) Pollinators as Bioindicators of the State of the Environment: Species, Activity and Diversity. Agriculture, Ecosystems and Environment, 74, 373-393. http://dx.doi.org/10.1016/S0167-8809(99)00044-4 
[6] Grissell, E. (2001) Insects and Gardens. Timber Press, Portland.

[7] Matteson, K.C., Ascher, J.S. and Langellotto, G.A. (2008) Bee Richness and Abundance in New York City Urban Gardens. Annals of the Entomological Society of America, 101, 140. http://dx.doi.org/10.1603/0013-8746(2008)101[140:BRAAIN]2.0.CO;2

[8] Bown, D. (1995) The Royal Horticultural Society Encyclopedia of Herbs \& Their Uses. Dorling Kindersley Ltd., London.

[9] Lorenzi, H. and de Souza, H.M. (2008) Ornamental Plants in Brazil: Shrubs, Herbs and Vines. 4th Edition, Instituto Nova Plantarum, Nova Odessa.

[10] IBGE_-Brazilian Institute of Geography and Statistics (2010) Santa Catarina. http://www.ibge.gov.br/estadosat/perfil.php?sigla=sc

[11] Gonçalves, L.S. (2012) Consequences of the Colony Collapse Disorder (CCD) of Bees in the International Beekeeping Agribusiness and Specially in Brazil. Proceedings of the X Meeting about Bees, Ribeirão Preto, 25-28 July 2012, 2425.

[12] Laroca, S., Cure, J.R. and Bortoli, C. (1982) The Association of Wild Bees (Hymenoptera, Apoidea) of a Restricted Area in the City of Curitiba (Brasil): A Biocoenotic Approach. Dusenia, 13, 93-117.

[13] Taura, H.M. (1990) The Community of Wild Bees (Hymenoptera, Apoidea) of Passeio Público, Curitiba, Paraná, Southern Brazil: A Comparative Approach. Master’s Dissertation, Parana Federal University, Curitiba.

[14] Knoll, F.R.N., Bego, L.R. and Imperatriz-Fonseca, V.L. (1994) The Bees in Urban Areas. In: Pirani Jr., J.R. and Cortopassi-Laurino, M., Eds., Flowers and Bees in São Paulo, 2nd Edition, EDUSP, São Paulo, 31-42.

[15] Taura, H.M. and Laroca, S. (1991) Highly Social Bees (Apidae) of a Restricted Area in Curitiba (Brazil): Distribution of Nests and Relative Abundance. Acta Biologica Parananense, 20, 85-101.

[16] Agostini, K. and Sazima, M. (2003) Ornamental Plants as Resources for Bees at the Campus of Campinas State University, São Paulo, Brazil. Bragantia, 62, 335-343.

[17] Salomé, J.A. (2002) Survey and Phenology of Bee Plants of Santa Catarina State. Master's Dissertation, Santa Catarina Federal University, Florianópolis.

[18] Salomé, J.A. and Orth, A.I. (2003) Santa Catarina’s Bee Plants and Their Action over the Hives. Mensagem Doce, 71, 12-16. http://www.apacame.org.br/mensagemdoce/71/artigo3.htm

[19] Salomé, J.A. and Orth, A.I. (2004) Diversity of the Bee Plants of Santa Catarina. Revista Agropecuária Catarinense, 17, 84-88.

[20] Frankie, G.W., Thorp, R.W., Hernandez, J., Rizzardi, M., Ertter, B., Pawelek, J.C., Witt, S.L., Schindler, M., Coville, R. and Wojcik, V.A. (2009) Native Bees Are a Rich Natural Resource in Urban California Gardens. California Agriculture, 63, 113-120. http://dx.doi.org/10.3733/ca.v063n03p113

[21] De Lima, H.C., Queiroz, L.P., Morim, M.P., Dutra, V.F., Bortoluzzi, R.L.C., Iganci, J.R.V., Fortunato, R.H., Vaz, A.M.S.F., De Souza, E.R., Filardi, F.L.R., Garcia, F.C.P., Fernandes, J.M., Martins-Da-Silva, R.C.V., Perez, A.P.F., Mansano, V.F., Miotto, S.T.S., Lima, L.C.P., Oliveira, M.L.A.A., Flores, A.S., Torke, B.M., Pinto, R.B., Lewis, G.P., Barros, M.J.F., Schütz, R., Pennington, T., Klitgaard, B.B., Rando, J.G., Scalon, V.R.., Da Costa, L.C., Da Silva, M.J., Moura, T.M., De Barros, L.A.V., Silva, M.C.R., Queiroz, R.T., Sartori, A.L.B., Camargo, R.A., Lima, I.B., Costa, J., Soares, M.V.B., Snak, C., Valls, J.F.M., São-Mateus, W., Falcão, M.J., Cardoso, D.B.O.S., Tozzi, A.M.G.A., Martins, M.V., Souza, V.C., Meireles, J.E. and Reis, I.P. (2013) Fabaceae. In: Lista de Espécies da Flora do Brasil, Jardim Botânico do Rio de Janeiro. http://floradobrasil.jbrj.gov.br/jabot/floradobrasil/FB115

[22] Ohashi, K. and Yahara, T. (2001) Behavioural Responses of Pollinators to Variation in Floral Display Size and Their Influence on the Evolution of Floral Traits. In: Chittka, L. and Thomson, J.D., Eds., Cognitive Ecology of Pollination, Cambridge University Press, Cambridge, 274-296. http://dx.doi.org/10.1017/CBO9780511542268.015

[23] Nakajima, J., Loeuille, B., Heiden, G., Dematteis, M., Hattori, E.K.O., Magenta, M.A.G., Ritter, M.R., Mondin, C.A., Roque, N., Ferreira, S.C., Borges, R.A.X., Soares, P.N., Almeida, G., Schneider, A., Sancho, G., Saavedra, M.M., Liro, R.M., Pereira, A.C.M., Moraes, M.D., Silva, G.A.R., Medeiros, J.D., Lorencini, T.S., Teles, A.M., Monge, M., Siniscalchi, C.M., Souza-Buturi, F.O., Bringel Jr., J.B.A., Carneiro, C.R., Pasini, E. and Oliveira, C.T. (2013) Asteraceae. In: Lista de Espécies da Flora do Brasil, Jardim Botânico do Rio de Janeiro. http://floradobrasil.jbri.gov.br/jabot/floradobrasil/FB55

[24] Matheson, A., Buchmann, S.L., O’Toole, C., Westrich, P., Williams, J.H. and Corbet, S.A. (1996) Which Bees Do Plants Need? In: Matheson, A., Buchmann, S.L., O’Toole, C., Westrich, P. and Williams, J.H., Eds., The Conservation of Bees, Academic Press, London, 105-114.

[25] Stehmann, J.R., Mentz, L.A., Agra, M.F., Vignoli-Silva, M., Giacomin, L. and Rodrigues, I.M.C. (2013) Solanaceae. In: Lista de Espécies da Flora do Brasil, Jardim Botânico do Rio de Janeiro. http://floradobrasil.jbri.gov.br/jabot/floradobrasil/FB225 
[26] Michener, C.D. (2007) The Bees of the World. 2nd Edition, John Hopkins University Press, Baltimore.

[27] Faegri, K. and Van Der Pijl, L. (1980) Principles of Pollination Ecology. Pergamon Press Ltd., Oxford.

[28] Frankie, G.W., Thorp, R.W., Schindler, M., Hernandez, J., Ertter, B. and Rizzardi, M. (2005) Ecological Patterns of Bees and Their Host Ornamental Flowers in Two Northern California Cities. Journal of the Kansas Entomoogical Society, 78, 227-246. http://dx.doi.org/10.2317/0407.08.1

[29] Lorenzi, H. (2013) Plants for Gardens in Brazil: Herbs, Shrubs and Vines. Instituto Plantarum, Nova Odessa.

[30] Souza, V.C., Cortopassi-Laurino, M., Simão-Bianchini, R., Pirani, J.R., Azoubel, M.L., Guibu, L.S. and Giannini, T.C. (1994) Bees Plants of São Paulo and Surroundings. In: Pirani, J.R. and Cortopassi-Laurino, M., Eds., Flowers and Bees at São Paulo, 2nd Edition, EDUSP, São Paulo, 43-67.

[31] Fenster, C.B., Armbruster, W.S., Wilson, P., Dudash, M.R. and Thomson, J.D. (2004) Pollination Syndromes and Floral Specialization. Annual Review of Ecology, Evolution, and Systematics, 35, 375-403. http://dx.doi.org/10.1146/annurev.ecolsys.34.011802.132347

[32] Cane, J.H., Johnson, E.A. and Klemens, M.W. (2005) Bees, Pollination and the Challenges of Sprawl. In: Johnson, E. and Klemens, M., Eds., Nature in Fragments: The Legacy of Sprawl, Columbia University Press, New York, 109-124.

[33] Cane, J.H. and Kervin, L. (2013) Gardening for Native Bees in Utah and Beyond. http://www.ars.usda.gov/SP2UserFiles/Place/20800500/Gardening.pdf

[34] Wojcik, V.A., Frankie, G.W., Thorp, R.W. and Hernandez, J. (2008) Seasonality in Bees and Their Floral Resource Plants at a Constructed Urban Bee Habitat in Berkeley, California. Journal of the Kansas Entomological Society, 81, 15-28. http://dx.doi.org/10.2317/JKES-701.17.1

[35] Althaus-Ottmann, M.M., Fogaça, L.A., Borsatto, R.S., Zuffellato-Ribas, K.C., Koehler, H.S. and Fonte, N.N. (2008) Why Study the Production of Ornamental Plants? The Santa Catarina's Case. Revista Brasileira de Horticultura Ornamental, 14, 1-6.

[36] Eremeeva, N.I. and Sushchev, D.V. (2005) Structural Changes in the Fauna of Pollinating Insects in Urban Landscapes. Russian Journal of Ecology, 36, 259-265. http://dx.doi.org/10.1007/s11184-005-0070-6

[37] Lorenzi, H. (1998) Brazilian Trees: Manual of Identification and Cultivation of Arboreal Plants of Brazil. 2nd Edition, Plantarum, Nova Odessa.

[38] Langellotto, G. (2013) Plants for Pollinators: Nurseries Can Highlight the Ecological Benefits of Pollinator-Friendly Plants. Digger, 5, 33-37.

[39] Hernandez, J.L., Frankie, G.W. and Thorp, R.W. (2009) Ecology of Urban Bees: A Review of Current Knowledge and Directions for Future Study. Cities and the Environment, 2, 1-15.

[40] Zadegan, Y.H., Behe, B.K. and Gough, R. (2008) Consumer Preferences for Native Plants in Montana Residential Landscapes and Perceptions for Naturalistic Designs. Journal of Environmental Horticulture, 26, 109-114.

[41] Allen-Wardell, G., Bernhardt, P., Bitner, R., Bitner, R., Burquez, A., Buchmann, S., Cane, J., Cox, P.A., Dalton, V., Feinseinger, P., Ingram, M., Inouye, D., Jones, C.E., Kennedy, K., Kevan, P., Koopowitz, H., Medellin, R., MedellinMorales, S., Nabhan, G.P., Pavlik, B., Tepedino, V., Torchio, P. and Walker, S. (1998) The Potential Consequences of Pollinator Declines on the Conservation of Biodiversity and Stability of Food Crop Yields. Conservation Biology, 12, 8-17. http://dx.doi.org/10.1046/j.1523-1739.1998.97154.x 


\section{Appendix: References of Table 1}

1 Cardoso Sobrinho, G. (2004) Bees and Wasps (Hymenoptera, Aculeata) in Areas Degraded by Open Coal Mining at Siderópolis, SC. Master's Dissertation, University of the Extreme South of Santa Catarina, Criciúma.

2 Feja, E.P. (2003) Wild Bees (Hymenoptera, Apoidea) of the Island of Santa Catarina: An Initial Survey of Wealth, Biological Aspects and Relations with Melittophilous Plants. Monography, Federal University of Santa Catarina, Florianópolis.

3 Figueiredo, G.C.N. (2003) The Diversity of Bees and Solitary Wasps Nesting in Trap-Nests (Hymenoptera, Aculeata) in the Aparados da Sierra National Park in Santa Catarina Territory. Scientific Initiation Report, University of the Extreme South of Santa Catarina, Criciúma.

4 Kamke, R. (2006) Diversity of Bees (Hymenoptera, Apoidea) in an Area of Sandbank in the State Park of Serra do Tabuleiro, Palhoca, SC. Monography, Federal University of Santa Catarina, Florianópolis.

5 Krug, C. (2007) The Community of Bees (Hymenoptera, Apiformes) of the Araucaria Forest in the Municipality of Porto União-SC and Bees as Floral Visitors of the Gourd (Cucurbita L.) in Santa Catarina, with Notes on Peponapis Fervens (Eucerini, Apidae). M.Sc. Dissertation, University of the Extreme South of Santa Catarina, Criciúma.

6 Luz, D.R. and Barroso, G.V. (2008) The Community of Bees (Hymenoptera, Apidae) and Its Floral Sources in the Serra do Itajaí National Park, Santa Catarina, Brazil. Proceedings of the VIII Meeting about Bees, Ribeirão Preto, Brazil, 23-26 July 2008, 642.

7 Mouga, D.M.D.S. (2004) The Community of Bees (Hymenoptera, Apoidea) in the Atlantic Rain Forest in Northeastern State of Santa Catarina, Brazil. Ph.D. Thesis, University of São Paulo, São Paulo.

8 Mouga, D.M.D.S. and Krug, C. (2010) Native Bee Communities (Apidae) in Montane Dense Rain Forest in Santa Catarina. Zoologia, 2, 70-80. http://dx.doi.org/10.1590/S1984-46702010000100011

9 Orth, A.I. (1983) Ecological Study of Wild Bees (Hymenoptera, Apoidea) at Caçador, SC, with Emphasis on Potential Pollinators of the Apple Tree Pyrus malus L. (Rosaceae). M.Sc. Dissertation, Federal University of Parana, Curitiba.

10 Ortoloan, S.M.L.S. (1989) Biocoenotics in Apoidea (Hymenoptera) of Apple Trees (Pyrus malus L.) at Lages, Santa Catarina, with Comparative Notes and Preliminary Pollination Experiment with Plebeia emerina. M.Sc. Dissertation, Federal University of Parana, Curitiba.

11 Ortolan, S.M.L.S. and Laroca, S. (1996) Melissocenotics in Apple Trees Growing Areas (Pyrus malus L.) in Lages (Santa Catarina, Southern Brazil), with Comparative Notes and Pollination Experiment with Plebeia emerina (Friese) (Hymenoptera, Apoidea). Acta Biológica Paranaense, 25, 1-113.

12 Sazan, M.S. and Alves-Dos-Santos, I. (2005) Bees and Wasps (Hymenoptera, Aculeata) Breeding in Nests Traps in the Ecological Park José Milaneze, Criciuma, SC. Scientific Initiation Report, University of the Extreme South of Santa Catarina, Criciúma.

13 Silva, M. (2005) Bees and Honey Plants at the Rural Zone of the Municipalities of Cocal do Sul, Criciuma and Nova Veneza, Located in the Coal Zone in the Southern State of Santa Catarina. M.Sc. Dissertation, University of the Extreme South of Santa Catarina, Criciúma.

14 Souza, T. (2005) Bees and Wasps (Hymenoptera, Aculeata) Breeding in Trap Nests at the Maracajá Municipal Park, SC. Scientific Initiation Report, University of the Extreme South of Santa Catarina, Criciúma.

15 Souza, T., Figueiredo, G.C.N. and Alves-Dos-Santos, I. (2005) Bees Visitors of Duranta repens Flowers (Verbenaceae) on the Campus of UNESC, Criciuma (SC). Scientific Initiation Report, University of the Extreme South of Santa Catarina, Criciúma.

16 Steiner, J., Harter-Marques, B., Zillikens, A. and Feja, E.P. (2006) Bees of Santa Catarina Island, Brazil—A First Survey and Checklist (Insect: Apoidea). Zootaxa, 1220, 1-18.

17 Wiese, H. (coord) (1987) New Apiculture. 8th Edition, Agropecuária, Porto Alegre.

18 Krug, C. (2010) The Community of Bees (Hymenoptera, Apiformes) in Two Areas of Ecological and Historical Interest in Santa Catarina. Ph.D. Thesis, University of São Paulo, São Paulo.

19 Mouga, D.M.D.S. and Dec, E. (2012) Pollen Catalog of Bee Medicinal Plants. DIOESC, Florianópolis.

20 Steiner, J., Zillikens, A., Kamke, R., Pickbrenner, E., Feja, E.P. and Falkenberg, D.B. (2010) Bees and Melittophilous Plants of Secondary Atlantic Forest Habitats at Santa Catarina Island, Southern Brazil. Oecologia Australis, 14, 16-39. http://dx.doi.org/10.4257/oeco.2010.1401.01

21 Mouga, D.M.D.S., Valentini, V.A., Krug, C., Anni, I.A.S. and Christovão, H.R. (2008) Native Bees Communities in Montane Dense Rain Forest (FODM) at the Environmental Preservation Area (APA) Rio Vermelho, at São Bento do Sul, SC. Proceedings of the XXVII Brazilian Congress of Zoology, Curitiba, 17-21 February 2008, 239.

22 Mouga, D.M.D.S., Dec, E., Warkentin, M. and Santos, A.K.G. (2014) Bee Communities and Floral Resources in a 
Temperate Area in Southern Brazil: A Descriptive of São Joaquim National Park, SC. In: Castilho, P.V., Omena, M.T.R.N. and Taniwaki, M.H., Eds., São Joaquim National Park: The Gateway of Knowledge, Editora Santarém, São Paulo, 135-163.

23 Dec, E. and Mouga, D.M.D.S. (2014) Diversity of Bees (Hymenoptera: Apidae) in an Atlantic Rain Forest Area at Joinville, Santa Catarina. Acta Biologica Catarinense, 1, 15-27.

24 Mouga, D.M.D.S. and Nogueira Neto, P. (2012) A High Grassland Bee Community in Southern Brazil: Survey and Annotated Checklist (Insecta: Apidae). Journal of the Kansas Entomological Society, 85, 295-308. http://dx.doi.org/10.2317/0022-8567-85.4.295

25 Mouga, D.M.D.S. and Nogueira-Neto, P. (2014) Community of Bees (Hymenoptera, Apidae) and Their Food Resources in Sandbanks and Atlantic Rain Forest at São Francisco do Sul, Santa Catarina, Brazil. In: Aguiar, A.J.C., Gonçalves, R.B. and Ramos, K.S., Eds., Essays on Bees of the Neotropical Region: Tribute to 80 Years of Danuncia Urban, Editora UFPR, Curitiba, in Press.

26 Mouga, D.M.D.S., Oliveira, D., Boeing, N.L. and Dec, E. (2012) Diversity of Bees in an Urban Area at Joinville, Santa Catarina, Brazil (Hymenoptera, Apidae). Proceedings of the XXIX Brazilian Congress of Zoology, Salvador, 5-9 March 2012, 1059.

27 Mouga, D.M.D.S., Warkentin, M., Santos, A.K.G., Dec, E., Silva, J.V., Barbosa, R.N. and Sena, J.C. (2013) Diversity of Apifauna and Bee Plants: Plant-Bee Interactions at the Ecological Station of Bracinho-Joinville/SC. Proceedings of the XI Congress of Ecology of Brazil \& I International Congress of Ecology, Porto Seguro, 15-19 September 2013, 306.

28 Warkentin, M., Ferretti, V., Baran, T.B.M., Santos, A.K.G., Barbosa, R.N. and Mouga, D.M.D.S. (2014) Bee Fauna Associated to Mangrove Plants in the Region of Babitonga Bay (Santa Catarina). Proceedings of the XXV Brazilian Congress of Entomology, Goiânia, 14-18 September 2014, 367. 